\title{
REES ALGEBRAS OF SQUARE-FREE MONOMIAL IDEALS
}

\author{
LOUIZA FOULI AND KUEI-NUAN LIN
}

\begin{abstract}
We determine the defining equations of the Rees algebra of an ideal $I$ in the case where $I$ is a squarefree monomial ideal such that each connected component of the line graph of the hypergraph corresponding to $I$ has at most 5 vertices. Moreover, we show in this case that the non-linear equations arise from even closed walks of the line graph, and we also give a description of the defining ideal of the toric ring when $I$ is generated by square-free monomials of the same degree. Furthermore, we provide a new class of ideals of linear type. We show that when $I$ is a square-free monomial ideal with any number of generators and the line graph of the hypergraph corresponding to $I$ is the graph of a disjoint union of trees and graphs with a unique odd cycle, then $I$ is an ideal of linear type.
\end{abstract}

1. Introduction. The main problem of interest in this article is the question of determining the defining equations of the Rees algebra of a square-free monomial ideal in a polynomial ring over a field. Let $R$ be a Noetherian ring, and let $I$ be an ideal. The Rees algebra, $\mathcal{R}(I)$, is defined to be the graded algebra

$$
\mathcal{R}(I)=R[I t]=\underset{i \geq 0}{\oplus} I^{i} t^{i} \subset R[t],
$$

where $t$ is an indeterminate. The Rees algebra of an ideal encodes many of the analytic properties of the variety defined by $I$, and it is the algebraic realization of the blowup of a variety along a subvariety. The blowup of $\operatorname{Spec}(R)$ along $V(I)$ is the projective spectrum of the

2010 AMS Mathematics subject classification. Primary 13A02, 13A15, 13A30, 05C05, 05E40, 05E45.

Keywords and phrases. Rees algebras, square-free monomial ideals, relation type, ideals of linear type, graph.

The first author was partially supported by a grant from the Simons Foundation, grant no. 244930 .

Received by the editors on August 30, 2013, and in revised form on January 29, 2014. 
Rees algebra, $\mathcal{R}(I)$, of $I$. This important construction is the main tool in the resolution of singularities of an algebraic variety.

From the algebraic point of view the Rees algebra of an ideal facilitates the study of the asymptotic behavior of the ideal, and it is essential in computing the integral closure of powers of ideals. The Rees algebra of an ideal $I$ in a Noetherian ring can be realized as a quotient of a polynomial ring, and hence once the defining ideal of $\mathcal{R}(I)$ is determined, it is straightforward to compute the integral closure, $\overline{\mathcal{R}(I)}$. It is well known that

$$
\overline{\mathcal{R}(I)}=\bigoplus_{i \geq 0} \overline{I^{i}} t^{i},
$$

and one obtains $\overline{I^{i}}=[\overline{\mathcal{R}(I)}]_{i}$.

Another reason for exploring the Rees algebra of an ideal is to determine the defining ideal of toric rings. Recall that a toric ideal is the ideal of relations of a monomial subring of a polynomial ring, see Section 2 for a detailed definition. For a Noetherian local ring $R$ and $I$ an $R$-ideal the special fiber ring, $\mathcal{F}(I)$, of $I$ is defined to be $\mathcal{F}(I)=\mathcal{R}(I) / I \mathcal{R}(I) \otimes k$, where $k$ is the residue field of $R$. When $R$ is a polynomial ring over a field $k$ and $I$ is generated by square-free monomials of the same degree, then the defining ideal of the special fiber ring $\mathcal{F}(I)$ is the toric ideal associated to $I$. Notice that in this case $\mathcal{F}(I)=k\left[f_{1}, \ldots, f_{n}\right]$, where $f_{1}, \ldots, f_{n}$ is a minimal monomial generating set of $I$. There are many questions arising from the study of toric varieties, as these have applications in combinatorics and in geometry, for example, see $[\mathbf{5}, \mathbf{7}, \mathbf{8}, \mathbf{2 7}, \mathbf{2 8}]$. Once we understand $\mathcal{R}(I)$ then we can obtain a concrete description of the toric ring corresponding to a square-free monomial ideal generated in the same degree directly from $\mathcal{R}(I)$.

The problem of finding an explicit description of the defining ideal of the Rees algebra has been addressed by many authors, see for example, $[2,4,9,10,11,12,13,14,21,25,26,29,34]$. In general, finding the generators for the defining ideal of the Rees algebra of an ideal is difficult. Recently, Kustin, Polini, and Ulrich in [21] studied the defining equations of the Rees algebra of certain height 2 ideals generated by forms of the same degree in $k[x, y]$, where $k$ is any field. Recently, Lin and Polini have extended these results in 
[23]. Furthermore, in [6], Cox, et al. provide a detailed study of the singularities of rational curves in $\mathbb{P}^{2}$ by studying the defining equations of the Rees algebra.

We consider the following construction for the Rees algebra. Let $R$ be a Noetherian ring, and let $I$ be an $R$-ideal. Let $f_{1}, \ldots, f_{n}$ be a minimal generating set for $I$. Consider the polynomial ring $S=R\left[T_{1}, \ldots, T_{n}\right]$, where $T_{1}, \ldots, T_{n}$ are indeterminates. Then there is a natural map $\phi: S \rightarrow \mathcal{R}(I)=R[I t]$ that sends $T_{i}$ to $f_{i} t$. Let $J=\operatorname{ker} \phi$ be the defining ideal of $\mathcal{R}(I)$. Then $\mathcal{R}(I) \simeq S / J$ and

$$
J=\bigoplus_{i=1}^{\infty} J_{i}
$$

is a graded ideal. A minimal generating set for $J$ is often referred to as the defining equations of the Rees algebra. Also, $J_{1}$ is known as the ideal of linear relations as it is the defining ideal of the symmetric algebra, $\operatorname{Sym}(I)$, of $I$ and it is generated by linear forms in the $T_{i}$. The generators of $J_{1}$ arise from the first syzygies of $I$. When $J=J_{1}$, then $I$ is called an ideal of linear type. In this case, $\mathcal{R}(I) \simeq \operatorname{Sym}(I)$, and the defining ideal of $\mathcal{R}(I)$ is well understood. Furthermore, if $R$ is a Noetherian local ring with residue field $k$ or a $k$-algebra where $k$ is a field, then there is a homomorphism $\psi: k\left[T_{1}, \ldots, T_{n}\right] \rightarrow \mathcal{F}(I)$ that sends $T_{i}$ to $f_{i}$. Then $\mathcal{F}(I) \simeq k\left[T_{1}, \ldots, T_{n}\right] / H$, where $H=\operatorname{ker} \psi$. An ideal $I$ is called an ideal of fiber type if the defining ideal $J$ of $\mathcal{R}(I)$ is obtained by the linear relations and the defining equations for the special fiber ring, i.e., $J=S J_{1}+S H$.

In the case of square-free monomial ideals generated in degree 2, namely, edge ideals, Villarreal showed that they are all ideals of fiber type, [35, Theorem 3.1]. Moreover, Villarreal gave an explicit description of the defining equations of the Rees algebra of any edge ideal, [35, Theorem 3.1]. It is also worth noting that Villarreal exhibited an example to show that his techniques do not extend for monomial ideals generated in degree higher than 2, [35, Example 3.1]. His example is a square-free monomial ideal generated in degree 3 that is not of fiber type. We remark that, in general, a square-free monomial ideal generated in degree greater than or equal to 3 is not necessarily of fiber type. Therefore, as the degree of the generators exceeds 2 , the complexity of the problem increases. 
In Section 2, we develop a series of lemmas that allow us to determine conditions under which an element in the defining ideal of the Rees algebra becomes redundant and hence not needed in the defining equations. We make use of a classic result by Taylor, in which she determines a non-minimal generating set for the defining ideal of the Rees algebra of any monomial ideal, [31]. We construct a graph associated to monomial ideals, which is also known as the line graph of the corresponding hypergraph. The monomial generators are the vertices for this graph, and the edges are determined by the greatest common divisor among the generators, see Construction 2.16. In the main result in this section we show that when $I$ is a squarefree monomial ideal such that the line graph of the corresponding hypergraph is the disjoint union of graphs with at most 5 vertices, then one can describe the defining equations of the Rees algebra of $I$, Theorem 2.20. Furthermore, we show that the non-linear part of the defining equations of $\mathcal{R}(I)$ arises from even closed walks of the line graph of the corresponding hypergraph, Theorem 2.20. Moreover, in Example 2.22, we give a concrete explanation of how one obtains the defining equations for the ideal in [35, Example 3.1].

As mentioned already, one can determine the toric ideal of a squarefree monomial ideal generated in the same degree directly from the defining equations of the Rees algebra. Villarreal provided a concrete description of the toric ideal in the case of square-free monomials generated in degree 2, [35, Proposition 3.1]. We are then able to give a generalization of Villarreal's result in the case of square-free monomial ideals generated in any degree, Corollary 2.23.

In Section 3, we concentrate on the special class of ideals of linear type. The first well-known class of ideals of linear type are complete intersection ideals, [24]. Many authors have worked on establishing other classes of ideals of linear type, see for example, $[\mathbf{1 5}, \mathbf{1 6}, \mathbf{1 7}$, $19,20,33]$. In the case of square-free monomial ideals generated in degree 2, Villarreal gave a complete characterization of ideals of linear type. More precisely, he showed that such an ideal $I$ is of linear type if and only if the graph of $I$ is the disjoint union of graphs of trees and graphs that have a unique odd cycle, [35, Corollary 3.2]. Inspired by this result, we prove that when $I$ is an ideal generated by squarefree monomials and the line graph of the hypergraph corresponding to $I$ is a disjoint union of graphs of trees and graphs with a unique 
odd cycle then $I$ is an ideal of linear type, Theorem 3.4. In some sense, these results extend the work of Villarreal, even though we do not fully recover his results. Nonetheless, our techniques allow us to consider square-free monomial ideals without any restrictions on the degrees of the generators nor any restrictions on the number of minimal generators.

We conclude with some final remarks. In [3], Conca and De Negri introduced the notion of monomial ideals generated by $M$-sequences. They showed that all ideals generated by an $M$-sequence are ideals of Gröbner linear type and, in particular, of linear type. In [30], Soleyman and Zheng introduced the class of monomial ideals of forest type and showed that in the case of square-free monomial ideals the class of monomial ideals of forest type coincides with the class of monomial ideals generated by $M$-sequences. It is straightforward to see that an ideal whose line graph is a graph of a forest is an ideal of forest type and hence making it an ideal generated by an $M$-sequence. Hence, by [3, Theorem 2.4], these ideals are of (Gröbner) linear type. However, an ideal whose line graph is the graph of an odd cycle is not an ideal of forest type, but nonetheless it is an ideal of linear type. Hence, the class of ideals of linear type that we uncovered in Theorem 3.4 are not necessarily generated by $M$-sequences, and thus our result is incomparable to the results of Conca and De Negri in [3].

2. The defining equations of the Rees algebra. Let $R$ be a polynomial ring over a field, and let $I$ be a monomial ideal in $R$. Let $f_{1}, \ldots, f_{n}$ be a minimal monomial generating set of $I$, and let $\mathcal{R}(I)$ denote the Rees algebra of $I$. Then $\mathcal{R}(I)=R\left[f_{1} t, \ldots, f_{n} t\right] \subset R[t]$, and there is an epimorphism $\phi: S=R\left[T_{1}, \ldots, T_{n}\right] \rightarrow \mathcal{R}(I)$ induced by $\phi\left(T_{i}\right)=f_{i}$ t. Let $J=\operatorname{ker} \phi$. We note that

$$
J=\bigoplus_{i=1}^{\infty} J_{i}
$$

is a graded ideal of $S$. As mentioned in the Introduction, a minimal generating set for the ideal $J$ is referred to as the defining equations of the Rees algebra of $I$.

Definition 2.1. Let $I$ be a monomial ideal in a polynomial ring $R$ over a field $k$. Let $f_{1}, \ldots, f_{n}$ be a minimal monomial generating set 
of $I$. Let $\mathcal{I}_{s}$ denote the set of all non-decreasing sequences of integers $\alpha=\left(i_{1}, \ldots, i_{s}\right) \subset\{1, \ldots, n\}$ of length $s$. Then $f_{\alpha}=f_{i_{1}} \cdots f_{i_{s}}$ is the corresponding product of monomials in $I$. We also let $T_{\alpha}=T_{i_{1}} \cdots T_{i_{s}}$ be the corresponding product of indeterminates in $S=R\left[T_{1}, \ldots, T_{n}\right]$. For every $\alpha, \beta \in \mathcal{I}_{s}$ we consider the binomial

$$
T_{\alpha, \beta}=\frac{f_{\beta}}{\operatorname{gcd}\left(f_{\alpha}, f_{\beta}\right)} T_{\alpha}-\frac{f_{\alpha}}{\operatorname{gcd}\left(f_{\alpha}, f_{\beta}\right)} T_{\beta} .
$$

The following is in [31], and we cite it here for ease of reference.

Theorem 2.2. ([31]). Let $R$ be a polynomial ring over a field, and let $I$ be a monomial ideal in $R$. Let $f_{1}, \ldots, f_{n}$ be a minimal monomial generating set of $I$, and let $\mathcal{R}(I)$ be the Rees algebra of $I$. Then $\mathcal{R}(I) \simeq S / J$, where $S=R\left[T_{1}, \ldots, T_{n}\right]$ is a polynomial ring, $T_{1}, \ldots, T_{n}$ are indeterminates, and

$$
J=S J_{1}+S \cdot\left(\bigcup_{i=2}^{\infty} J_{i}\right)
$$

such that $J_{s}=\left\{T_{\alpha, \beta} \mid\right.$ for $\left.\alpha, \beta \in \mathcal{I}_{s}\right\}$.

The following remark states some properties for the greatest common divisor among square-free monomials. The proofs are omitted as they are elementary.

Remark 2.3. Let $a, b, c, d, b_{1}, \ldots, b_{s}$ be square-free monomials in a polynomial ring $R$ over a field. Let $n, m, l, r$ be positive integers. Then

(a) $\operatorname{gcd}\left(a^{n}, b^{m}\right)=\operatorname{gcd}(a, b)^{\min \{n, m\}}$,

(b) $\operatorname{gcd}\left(a^{n}, b_{1} \cdots b_{s}\right)=\operatorname{gcd}\left(a^{s}, b_{1} \cdots b_{s}\right)$ for all $n \geq s$,

(c) $\operatorname{gcd}\left(a^{n}, b^{m} c^{l}\right)=\operatorname{gcd}(a, b)^{\min \{n, m\}} \operatorname{gcd}(a, c)^{\min \{n, l\}} / C$, for some $C \in$ $R$.

The main goal in this section is to determine conditions under which for sequences $\alpha, \beta$ of length $s \geq 2$ we have

$$
T_{\alpha, \beta} \in S J_{1}+S \cdot\left(\bigcup_{i=2}^{s-1} J_{i}\right) .
$$


In other words, we are interested in finding conditions on the sequences $\alpha, \beta$ such that the generator $T_{\alpha, \beta}$ is redundant in the defining ideal of the Rees algebra. The following two lemmas follow directly from Definition 2.1.

Lemma 2.4. Let $R$ be a polynomial ring over a field, and let $I$ be a monomial ideal in $R$. Let $\alpha, \beta \in \mathcal{I}_{s}$ be two sequences of length $s \geq 2$, where $\mathcal{I}_{s}$ is as in Definition 2.1. Suppose that $\alpha=\left(a_{1}, \ldots, a_{s}\right)$, $\beta=\left(b_{1}, \ldots, b_{s}\right)$, and suppose that $a_{i}=b_{j}$ for some $i$ and some $j$. Then

$$
T_{\alpha, \beta} \in S J_{1}+S \cdot\left(\bigcup_{i=2}^{s-1} J_{i}\right)
$$

Proof. Without loss of generality, we may assume that $a_{1}=b_{1}$. Let $\alpha_{1}=\left(a_{2}, \ldots, a_{s}\right)$ and $\beta_{1}=\left(b_{2}, \ldots, b_{s}\right)$. Then $\operatorname{gcd}\left(f_{\alpha}, f_{\beta}\right)=$ $f_{a_{1}} \operatorname{gcd}\left(f_{\alpha_{1}}, f_{\beta_{1}}\right)=f_{b_{1}} \operatorname{gcd}\left(f_{\alpha_{1}}, f_{\beta_{1}}\right)$. Notice that $f_{a_{1}}=f_{b_{1}}$ and $T_{a_{1}}=T_{b_{1}}$. Then

$$
\begin{aligned}
T_{\alpha, \beta} & =\frac{f_{\beta}}{\operatorname{gcd}\left(f_{\alpha}, f_{\beta}\right)} T_{\alpha}-\frac{f_{\alpha}}{\operatorname{gcd}\left(f_{\alpha}, f_{\beta}\right)} T_{\beta} \\
& =T_{a_{1}}\left[\frac{f_{\beta_{1}}}{\operatorname{gcd}\left(f_{\alpha_{1}}, f_{\beta_{1}}\right)} T_{\alpha_{1}}-\frac{f_{\alpha_{1}}}{\operatorname{gcd}\left(f_{\alpha_{1}}, f_{\beta_{1}}\right)} T_{\beta_{1}}\right] \\
& =T_{a_{1}}\left[T_{\alpha_{1}, \beta_{1}}\right] \in S J_{1}+S \cdot\left(\bigcup_{i=2}^{s-1} J_{i}\right)
\end{aligned}
$$

Lemma 2.5. Let $R$ be a polynomial ring over a field, and let $I$ be a monomial ideal in $R$. Let $\alpha, \beta \in \mathcal{I}_{s}$ be two sequences of length $s \geq 2$, where $\mathcal{I}_{s}$ is as in Definition 2.1. Suppose that $\alpha=\left(\alpha_{1}, \ldots, \alpha_{1}\right)$ and $\beta=\left(\beta_{1}, \ldots, \beta_{1}\right)$, where $\alpha_{1}, \beta_{1} \in \mathcal{I}_{m}$ and $m \leq s$. Then

$$
T_{\alpha, \beta} \in S J_{1}+S \cdot\left(\bigcup_{i=2}^{m} J_{i}\right) .
$$

Proof. First we observe that $s$ is a multiple of $m$. Let $l$ be an integer such that $s=l m$. Notice that $f_{\alpha}=f_{\alpha_{1}}^{l}$ and $f_{\beta}=f_{\beta_{1}}^{l}$. Also, by Remark 2.3 (a), we have that $\operatorname{gcd}\left(f_{\alpha}, f_{\beta}\right)=\operatorname{gcd}\left(f_{\alpha_{1}}, f_{\beta_{1}}\right)^{l}$. Let

$$
a=\frac{f_{\beta_{1}} T_{\alpha_{1}}}{\operatorname{gcd}\left(f_{\alpha_{1}}, f_{\beta_{1}}\right)}, \quad b=\frac{f_{\alpha_{1}} T_{\beta_{1}}}{\operatorname{gcd}\left(f_{\alpha_{1}}, f_{\beta_{1}}\right)},
$$


and let $A=\left(a^{l-1}+a^{l-2} b+\cdots+a b^{l-2}+b^{l-1}\right)$. Notice that $a, b \in S$, and thus $A \in S$. Then

$$
\begin{aligned}
T_{\alpha, \beta} & =\frac{f_{\beta}}{\operatorname{gcd}\left(f_{\alpha}, f_{\beta}\right)} T_{\alpha}-\frac{f_{\alpha}}{\operatorname{gcd}\left(f_{\alpha}, f_{\beta}\right)} T_{\beta}=a^{l}-b^{l} \\
& =(a-b)\left(a^{l-1}+a^{l-2} b+\cdots+a b^{l-2}+b^{l-1}\right) \\
& =T_{a_{1}, b_{1}} A \in S J_{1}+S \cdot\left(\bigcup_{i=2}^{m} J_{i}\right) .
\end{aligned}
$$

We observe the following properties for greatest common divisors among monomials. Again, we omit the proofs as they are elementary.

Remark 2.6. Let $a, b, c, d, e, f$ be monomials in a polynomial ring $R$ over a field.

(a) $\operatorname{gcd}(a, b c)=\operatorname{gcd}(a, b) \operatorname{gcd}(a, c) / C$, for some $C \in R$.

(b) Suppose that $\operatorname{gcd}(a, c)=1$. Then $\operatorname{gcd}(a b, c)=\operatorname{gcd}(b, c)$.

The following lemma plays an important role in the rest of this article as it allows us to show that certain expressions $T_{\alpha, \beta}$ are redundant in the defining ideal of the Rees algebra.

Lemma 2.7. Let $R$ be a polynomial ring over a field, and let $I$ be a monomial ideal in $R$. Let $\alpha, \beta \in \mathcal{I}_{s}$ be two sequences of length $s \geq 2$, where $\mathcal{I}_{s}$ is as in Definition 2.1. Suppose $\alpha=\left(\alpha_{1}, \ldots, \alpha_{m}\right)$ and $\beta=\left(\beta_{1}, \ldots, \beta_{m}\right)$ with $\alpha_{i}, \beta_{i} \in I_{s_{i}}$ and $s_{1}+\cdots+s_{m}=$ s. Suppose that, for all $1 \leq i \leq m$, there exist $C_{i} \in R$ such that

$$
\begin{aligned}
& \operatorname{gcd}\left(f_{\alpha}, f_{\beta}\right) \\
& =\operatorname{gcd}\left(\prod_{j=1}^{i-1} f_{\alpha_{j}}, f_{\beta}\right) \operatorname{gcd}\left(\prod_{k=i}^{m} f_{\alpha_{k}}, \prod_{k=i+1}^{m} f_{\beta_{k}}\right) \operatorname{gcd}\left(f_{\alpha_{i}}, f_{\beta_{i}}\right) / C_{i},
\end{aligned}
$$

where empty products are taken to be 1 . Then

$$
T_{\alpha, \beta}=\sum_{i=1}^{m}\left[A_{i} \prod_{j=i+1}^{m} T_{\alpha_{j}} \prod_{k=1}^{i-1} T_{\beta_{k}}\right] T_{\alpha_{i}, \beta_{i}} \in S J_{1}+S \cdot\left(\bigcup_{i=2}^{r} J_{i}\right),
$$

where $r=\max \left\{s_{1}, \ldots, s_{m}\right\}$ and $A_{i} \in R$ for all $i$. 
Proof. Notice that

$$
f_{\alpha}=\prod_{i=1}^{m} f_{\alpha_{i}} \quad \text { and } f_{\beta}=\prod_{i=1}^{m} f_{\beta_{i}} .
$$

Then we have

$$
\begin{aligned}
T_{\alpha, \beta}= & \frac{f_{\beta}}{\operatorname{gcd}\left(f_{\alpha}, f_{\beta}\right)} T_{\alpha}-\frac{f_{\alpha}}{\operatorname{gcd}\left(f_{\alpha}, f_{\beta}\right)} T_{\beta} \\
= & \sum_{i=1}^{m}\left[\frac{C_{i} \prod_{j=1}^{i-1} f_{\alpha_{j}} \prod_{k=i+1}^{m} f_{\beta_{k}} \prod_{j=i+1}^{m} T_{\alpha_{j}} \prod_{k=1}^{i-1} T_{\beta_{k}}}{\operatorname{gcd}\left(\prod_{j=1}^{i-1} f_{\alpha_{j}}, f_{\beta}\right) \operatorname{gcd}\left(\prod_{k=i}^{m} f_{\alpha_{k}}, \prod_{k=i+1}^{m} f_{\beta_{k}}\right)}\right. \\
& \left.\left(\frac{f_{\beta_{i}}}{\operatorname{gcd}\left(f_{\alpha_{i}}, f_{\beta_{i}}\right)} T_{\alpha_{i}}-\frac{f_{\alpha_{i}}}{\operatorname{gcd}\left(f_{\alpha_{i}}, f_{\beta_{i}}\right)} T_{\beta_{i}}\right)\right] .
\end{aligned}
$$

Finally, we note that

$$
A_{i}=\frac{C_{i} \prod_{j=1}^{i-1} f_{\alpha_{j}} \prod_{k=i+1}^{m} f_{\beta_{k}}}{\operatorname{gcd}\left(\prod_{j=1}^{i-1} f_{\alpha_{j}}, f_{\beta}\right) \operatorname{gcd}\left(\prod_{k=i}^{m} f_{\alpha_{k}}, \prod_{k=i+1}^{m} f_{\beta_{k}}\right)} \in R .
$$

Next we give a list of conditions that, when satisfied by two sequences $\alpha, \beta$, then the generator $T_{\alpha, \beta}$ is not a minimal generator in the defining ideal of the Rees algebra of the corresponding ideal.

Proposition 2.8. Let $R$ be a polynomial ring over a field, and let $I$ be a monomial ideal in $R$. Let $\alpha, \beta \in \mathcal{I}_{s}$ be two sequences of length $s \geq 2$, where $\mathcal{I}_{s}$ is as in Definition 2.1. Let $\alpha=\left(a_{1}, \ldots, a_{s}\right), \beta=\left(b_{1}, \ldots, b_{s}\right)$. Suppose that, after some reordering, there exist integers $k, l$ with $1 \leq k$, $l \leq s-1$ such that $\operatorname{gcd}\left(f_{a_{i}}, f_{b_{j}}\right)=1$ for every $1 \leq i \leq l$ and every $k+1 \leq j \leq s$. We further assume that $\operatorname{gcd}\left(f_{a_{u}}, f_{b_{v}}\right)=1$, for every $l+1 \leq u \leq s$ and every $1 \leq v \leq k$. Then

$$
T_{\alpha, \beta} \in S J_{1}+S \cdot\left(\bigcup_{i=2}^{r} J_{i}\right),
$$

where $r=\max \{k,|k-l|, s-k, s-l\}$. 
Proof. Suppose that $l=k$, and write $\alpha=\left(\alpha_{1}, \alpha_{2}\right)$ and $\beta=\left(\beta_{1}, \beta_{2}\right)$, where $\alpha_{1}=\left(a_{1}, \ldots, a_{k}\right), \alpha_{2}=\left(a_{k+1}, \ldots, a_{s}\right), \beta_{1}=\left(b_{1}, \ldots, b_{k}\right)$, and $\beta_{2}=\left(b_{k+1}, \ldots, b_{s}\right)$. By our assumptions, we have $\operatorname{gcd}\left(f_{\alpha_{i}}, f_{\beta_{j}}\right)=1$ for $i \neq j$. Hence, by Remark 2.6, we have

$$
\begin{aligned}
\operatorname{gcd}\left(f_{\alpha}, f_{\beta}\right) & =\operatorname{gcd}\left(f_{\alpha}, f_{\beta_{1}}\right) \operatorname{gcd}\left(f_{\alpha}, f_{\beta_{2}}\right) / C_{1} \\
& =\operatorname{gcd}\left(f_{\alpha_{1}}, f_{\beta_{1}}\right) \operatorname{gcd}\left(f_{\alpha}, f_{\beta_{2}}\right) / C_{1}, \\
\operatorname{gcd}\left(f_{\alpha}, f_{\beta}\right) & =\operatorname{gcd}\left(f_{\alpha_{1}}, f_{\beta}\right) \operatorname{gcd}\left(f_{\alpha_{2}}, f_{\beta}\right) / C_{2} \\
& =\operatorname{gcd}\left(f_{\alpha_{1}}, f_{\beta}\right) \operatorname{gcd}\left(f_{\alpha_{2}}, f_{\beta_{2}}\right) / C_{2} .
\end{aligned}
$$

The result follows from Lemma 2.7 with $m=2$.

Without loss of generality, we may now assume that $l<k$. We write $\alpha=\left(\alpha_{1}, \alpha_{2}, \alpha_{3}\right)$ and $\beta=\left(\beta_{1}, \beta_{2}, \beta_{3}\right)$, with $\alpha_{1}=\left(a_{1}, \ldots, a_{l}\right)$, $\alpha_{2}=\left(a_{l+1}, \ldots, a_{k}\right), \alpha_{3}=\left(a_{k+1}, \ldots, a_{s}\right), \beta_{1}=\left(b_{1}, \ldots, b_{l}\right), \beta_{2}=$ $\left(b_{l+1}, \ldots, b_{k}\right)$, and $\beta_{3}=\left(b_{k+1}, \ldots, b_{s}\right)$. Then $\operatorname{gcd}\left(f_{\alpha_{1}}, f_{\beta_{3}}\right)=1$ and $\operatorname{gcd}\left(f_{\alpha_{i}}, f_{\beta_{j}}\right)=1$ for $i=2,3$ and $j=1,2$. Hence by Remark 2.6, we have

$$
\begin{aligned}
\operatorname{gcd}\left(f_{\alpha}, f_{\beta}\right) & =\operatorname{gcd}\left(f_{\alpha}, f_{\beta_{1}}\right) \operatorname{gcd}\left(f_{\alpha}, f_{\beta_{2}} f_{\beta_{3}}\right) / C_{1} \\
& =\operatorname{gcd}\left(f_{\alpha_{1}}, f_{\beta_{1}}\right) \operatorname{gcd}\left(f_{\alpha}, f_{\beta_{2}} f_{\beta_{3}}\right) / / C_{1}, \\
\operatorname{gcd}\left(f_{\alpha}, f_{\beta}\right) & =\operatorname{gcd}\left(f_{\alpha_{1}}, f_{\beta}\right) \operatorname{gcd}\left(f_{\alpha_{2}} f_{\alpha_{3}}, f_{\beta}\right) / C_{2} \\
& =\operatorname{gcd}\left(f_{\alpha_{1}}, f_{\beta}\right) \operatorname{gcd}\left(f_{\alpha_{2}} f_{\alpha_{3}}, f_{\beta_{3}}\right) \operatorname{gcd}\left(f_{\alpha_{2}} f_{\alpha_{3}}, f_{\beta_{1}} f_{\beta_{2}}\right) / C_{2}^{\prime} \\
& =\operatorname{gcd}\left(f_{\alpha_{1}}, f_{\beta}\right) \operatorname{gcd}\left(f_{\alpha_{2}} f_{\alpha_{3}}, f_{\beta_{3}}\right) \operatorname{gcd}\left(f_{\alpha_{2}} f_{\alpha_{3}}, f_{\beta_{2}}\right) / C_{2}^{\prime} \\
& =\operatorname{gcd}\left(f_{\alpha_{1}}, f_{\beta}\right) \operatorname{gcd}\left(f_{\alpha_{2}} f_{\alpha_{3}}, f_{\beta_{3}}\right) \operatorname{gcd}\left(f_{\alpha_{2}}, f_{\beta_{2}}\right) / C_{2}^{\prime},
\end{aligned}
$$

and

$$
\begin{aligned}
\operatorname{gcd}\left(f_{\alpha}, f_{\beta}\right) & =\operatorname{gcd}\left(f_{\alpha_{1}} f_{\alpha_{2}}, f_{\beta}\right) \operatorname{gcd}\left(f_{\alpha_{3}}, f_{\beta}\right) / C_{3} \\
& =\operatorname{gcd}\left(f_{\alpha_{1}} f_{\alpha_{2}}, f_{\beta}\right) \operatorname{gcd}\left(f_{\alpha_{3}}, f_{\beta_{3}}\right) / C_{3} .
\end{aligned}
$$

Therefore, we may apply Lemma 2.7 with $m=3$.

The next three lemmas deal with possible repetitions in the sequences $\alpha, \beta \in \mathcal{I}_{s}$ and how that affects the term $T_{\alpha, \beta}$.

Lemma 2.9. Let $R$ be a polynomial ring over a field, and let $I$ be a square-free monomial ideal in $R$. Let $\alpha, \beta \in \mathcal{I}_{s}$ be two sequences of length $s \geq 2$, where $\mathcal{I}_{s}$ is as in Definition 2.1. Suppose that 
$\alpha=\left(a_{1}, \ldots, a_{1}\right)$ and $\beta=\left(b_{1}, \ldots, b_{s}\right)$. Then

$$
T_{\alpha, \beta} \in S J_{1}+S \cdot\left(\bigcup_{i=2}^{s-1} J_{i}\right) .
$$

Proof. Notice that $f_{\alpha}=f_{a_{1}}^{s}$ and $f_{\beta}=f_{b_{1}} \cdots f_{b_{s}}$. Let $\beta_{1}=$ $\left(b_{2}, \ldots, b_{s}\right)$. We also note that $\operatorname{gcd}\left(f_{a_{1}}^{s}, f_{b_{1}}\right)=\operatorname{gcd}\left(f_{a_{1}}, f_{b_{1}}\right)$, by Remark 2.3 (a). Then

$$
\begin{aligned}
\operatorname{gcd}\left(f_{\alpha}, f_{\beta}\right) & =\operatorname{gcd}\left(f_{a_{1}}^{s}, f_{b_{1}}\right) \operatorname{gcd}\left(f_{a_{1}}^{s}, f_{\beta_{1}}\right) / C \\
& =\operatorname{gcd}\left(f_{a_{1}}, f_{b_{1}}\right) \operatorname{gcd}\left(f_{a_{1}}^{s-1}, f_{\beta_{1}}\right) / C,
\end{aligned}
$$

for some $C \in R$, by Remark 2.3 (b) and (c). Hence,

$$
T_{\alpha, \beta} \in S J_{1}+S \cdot\left(\bigcup_{i=2}^{s-1} J_{i}\right),
$$

by Lemma 2.7 .

Lemma 2.10. Let $R$ be a polynomial ring over a field, and let $I$ be a square-free monomial ideal in $R$. Let $\alpha, \beta \in \mathcal{I}_{s}$ be two sequences of length $s \geq 2$, where $\mathcal{I}_{s}$ is as in Definition 2.1. Suppose that $\alpha=\left(a_{1}, \ldots, a_{1}, a_{2}, \ldots, a_{2}\right)$ and $\beta=\left(b_{1}, \ldots, b_{1}, b_{2}, \ldots, b_{2}\right)$. Then $T_{\alpha, \beta} \in S J_{1}+S J_{2}$.

Proof. We will proceed by induction on $s$. If $s=2$, then there is nothing to show. Suppose that $s>2$. Suppose that there are $l_{i}$ distinct copies of $a_{i}$ in $\alpha$ and $k_{i}$ distinct copies of $b_{i}$ in $\beta$ for $i=1,2$. Then $f_{\alpha}=f_{a_{1}}^{l_{1}} f_{a_{2}}^{l_{2}}$ and $f_{\beta}=f_{b_{1}}^{k_{1}} f_{b_{2}}^{k_{2}}$. For simplicity, we write $\alpha=\left(a_{1}^{l_{1}}, a_{2}^{l_{2}}\right)$ and $\beta=\left(b_{1}^{k_{1}}, b_{2}^{k_{2}}\right)$. If $k_{1}=k_{2}$ and $l_{1}=l_{2}$, then the result follows from Lemma 2.5. Thus, we may assume without loss of generality that $k_{1}>k_{2}$ and $l_{1} \geq l_{2}$. We will show

$$
\begin{aligned}
\operatorname{gcd}\left(f_{\alpha}, f_{\beta}\right) & =\operatorname{gcd}\left(f_{a_{1}}^{l_{1}-1} f_{a_{2}}^{l_{2}}, f_{b_{1}}^{k_{1}} f_{b_{2}}^{k_{2}}\right) \operatorname{gcd}\left(f_{a_{1}}, f_{b_{1}}\right) / G \\
& =\operatorname{gcd}\left(f_{a_{1}}^{l_{1}-1} f_{a_{2}}^{l_{2}}, f_{b_{1}}^{k_{1}-1} f_{b_{2}}^{k_{2}}\right) \operatorname{gcd}\left(f_{a_{1}}^{l_{1}} f_{a_{2}}^{l_{2}}, f_{b_{1}}\right) / H
\end{aligned}
$$

for some $G, H \in R$. Then $T_{\alpha, \beta} \in S J_{1} \cup S J_{2}$, by Lemma 2.7 and the induction. 
Notice that we have the following equalities:

$$
\begin{aligned}
& \operatorname{gcd}\left(f_{\alpha}, f_{\beta}\right) \\
& =\operatorname{gcd}\left(f_{a_{1}}^{l_{1}-1} f_{a_{2}}^{l_{2}}, f_{b_{1}}^{k_{1}} f_{b_{2}}^{k_{2}}\right) \operatorname{gcd}\left(f_{a_{1}}, f_{b_{1}}^{k_{1}} f_{b_{2}}^{k_{2}}\right) / C \\
& =\operatorname{gcd}\left(f_{a_{1}}^{l_{1}-1} f_{a_{2}}^{l_{2}}, f_{b_{1}}^{k_{1}} f_{b_{2}}^{k_{2}}\right) \operatorname{gcd}\left(f_{a_{1}}, f_{b_{1}}^{k_{1}-1} f_{b_{2}}^{k_{2}}\right) \operatorname{gcd}\left(f_{a_{1}}, f_{b_{1}}\right) / C D, \\
& \operatorname{gcd}\left(f_{\alpha}, f_{\beta}\right) \\
& =\operatorname{gcd}\left(f_{a_{1}}^{l_{1}} f_{a_{2}}^{l_{2}}, f_{b_{1}}^{k_{1}-1} f_{b_{2}}^{k_{2}}\right) \operatorname{gcd}\left(f_{a_{1}}^{l_{1}} f_{a_{2}}^{l_{2}}, f_{b_{1}}\right) / E \\
& =\operatorname{gcd}\left(f_{a_{1}}^{l_{1}-1} f_{a_{2}}^{l_{2}}, f_{b_{1}}^{k_{1}-1} f_{b_{2}}^{k_{2}}\right) \operatorname{gcd}\left(f_{a_{1}}, f_{b_{1}}^{k_{1}-1} f_{b_{2}}^{k_{2}}\right) \operatorname{gcd}\left(f_{a_{1}}^{l_{1}} f_{a_{2}}^{l_{2}}, f_{b_{1}}\right) / E F,
\end{aligned}
$$

for some $C, D, E, F \in R$ by Remark 2.6 (a). We will show that, for any integer $t$ and any variable $x \in R$, if $x^{t} \mid \operatorname{gcd}\left(f_{a_{1}}, f_{b_{1}}^{k_{1}} f_{b_{2}}^{k_{2}}\right)$, then $x^{t} \mid C D$ and $x^{t} \mid E F$. This is equivalent to showing that, for any variable $x \in R$ and any integer $u$, if $x \mid \operatorname{gcd}\left(f_{a_{1}}, f_{b_{1}}^{k_{1}} f_{b_{2}}^{k_{2}}\right)$ and $x^{u} \mid \operatorname{gcd}\left(f_{\alpha}, f_{\beta}\right)$ then $x^{u} \mid \operatorname{gcd}\left(f_{a_{1}}^{l_{1}-1} f_{a_{2}}^{l_{2}}, f_{b_{1}}^{k_{1}} f_{b_{2}}^{k_{2}}\right) \operatorname{gcd}\left(f_{a_{1}}, f_{b_{1}}\right)$ and $x^{u} \mid \operatorname{gcd}\left(f_{a_{1}}^{l_{1}-1} f_{a_{2}}^{l_{2}}, f_{b_{1}}^{k_{1}-1} f_{b_{2}}^{k_{2}}\right) \operatorname{gcd}\left(f_{a_{1}}^{l_{1}} f_{a_{2}}^{l_{2}}, f_{b_{1}}\right)$. This follows immediately since $s=l_{1}+l_{2}=k_{1}+k_{2}>l_{1}$ and $l_{1} \geq s / 2>k_{2}$.

Lemma 2.11. Let $R$ be a polynomial ring over a field, and let $I$ be a square-free monomial ideal in $R$. Let $\alpha, \beta \in \mathcal{I}_{s}$ be two sequences of length $s \geq 4$, where $\mathcal{I}_{s}$ is as in Definition 2.1. Suppose that $\alpha$ and $\beta$ are of the form $\alpha=\left(a_{1}, \ldots, a_{1}, a_{2}, \ldots, a_{2}, a_{3}, \ldots, a_{3}\right)$ and $\beta=\left(b_{1}, \ldots, b_{1}, b_{2}, \ldots, b_{2}\right)$. Then $T_{\alpha, \beta} \in S J_{1}+S \cdot\left(J_{2} \cup J_{3}\right)$.

Proof. We will show

$$
T_{\alpha, \beta} \in S J_{1}+S \cdot\left(\bigcup_{i=2}^{s-1} J_{i}\right)
$$

and by induction on $s \geq 4$, we conclude that $T_{\alpha, \beta} \in S J_{1}+S \cdot\left(J_{2} \cup J_{3}\right)$. Suppose that there are $l_{i}$ distinct copies of $a_{i}$ in $\alpha$ and $k_{i}$ distinct copies of $b_{i}$ in $\beta$. Then $l_{1}+l_{2}+l_{3}=k_{1}+k_{2}=s \geq 4$. Without lost of generality, we assume that $l_{1} \geq l_{2} \geq l_{3} \geq 1$ and $k_{1} \geq k_{2} \geq 1$. Since $s \geq 4$, we have $l_{1} \geq 2$ and $k_{1} \geq 2$. We write $\alpha=\left(a_{1}^{l_{1}}, a_{2}^{l_{2}}, a_{3}^{l_{3}}\right)$ and $\beta=\left(b_{1}^{k_{1}}, b_{2}^{k_{2}}\right)$. We then have three possible scenarios. We claim the following 
(i) If $l_{1}+l_{2}>k_{1}$, then

$$
\begin{aligned}
\operatorname{gcd}\left(f_{\alpha}, f_{\beta}\right) & =\operatorname{gcd}\left(f_{a_{1}} f_{a_{2}}, f_{b_{1}} f_{b_{2}}\right) \operatorname{gcd}\left(f_{\alpha_{1}}, f_{\beta}\right) / A \\
& =\operatorname{gcd}\left(f_{\alpha}, f_{b_{1}} f_{b_{2}}\right) \operatorname{gcd}\left(f_{\alpha_{1}}, f_{\beta_{1}}\right) / B,
\end{aligned}
$$

for some $A, B \in R$, and $\alpha_{1}=\left(a_{1}^{l_{1}-1}, a_{2}^{l_{2}-1}, a_{3}^{l_{3}}\right), \beta_{1}=\left(b_{1}^{k_{1}-1}, b_{2}^{k_{2}-1}\right)$.

(ii) If $l_{1}>k_{2}$, then

$$
\begin{aligned}
\operatorname{gcd}\left(f_{\alpha}, f_{\beta}\right) & =\operatorname{gcd}\left(f_{a_{1}}, f_{b_{1}}\right) \operatorname{gcd}\left(f_{\alpha_{1}}, f_{\beta}\right) / C \\
& =\operatorname{gcd}\left(f_{\alpha}, f_{b_{1}}\right) \operatorname{gcd}\left(f_{\alpha_{1}}, f_{\beta_{1}}\right) / D,
\end{aligned}
$$

for some $C, D \in R$, and $\alpha_{1}=\left(a_{1}^{l_{1}-1}, a_{2}^{l_{2}}, a_{3}^{l_{3}}\right), \beta_{1}=\left(b_{1}^{k_{1}-1}, b_{2}^{k_{2}}\right)$.

(iii) If $l_{1}+l_{2} \leq k_{1}$ and $l_{1} \leq k_{2}$, then

$$
\begin{aligned}
\operatorname{gcd}\left(f_{\alpha}, f_{\beta}\right) & =\operatorname{gcd}\left(f_{a_{1}}^{s / 3} f_{a_{2}}^{s / 3} f_{a_{3}}^{s / 3}, f_{b_{1}}^{2 s / 3} f_{b_{2}}^{s / 3}\right) \\
& =\left(\operatorname{gcd}\left(f_{a_{1}} f_{a_{2}} f_{a_{3}}, f_{b_{1}}^{2} f_{b_{2}}\right)\right)^{s / 3} .
\end{aligned}
$$

Once we obtain the above claims then the result will follow by Lemma 2.7.

To establish claim (i), we notice that we have the following equalities:

$$
\begin{aligned}
\operatorname{gcd}\left(f_{\alpha}, f_{\beta}\right) & =\operatorname{gcd}\left(f_{a_{1}} f_{a_{2}}, f_{\beta}\right) \operatorname{gcd}\left(f_{\alpha_{1}}, f_{\beta}\right) / E \\
& =\operatorname{gcd}\left(f_{a_{1}} f_{a_{2}}, f_{b_{1}} f_{b_{2}}\right) \operatorname{gcd}\left(f_{a_{1}} f_{a_{2}}, f_{\beta_{1}}\right) \operatorname{gcd}\left(f_{\alpha_{1}}, f_{\beta}\right) / E F, \\
& =\operatorname{gcd}\left(f_{\alpha}, f_{b_{1}} f_{b_{2}}\right) \operatorname{gcd}\left(f_{\alpha}, f_{\beta_{1}}\right) / G \\
& =\operatorname{gcd}\left(f_{\alpha}, f_{b_{1}} f_{b_{2}}\right) \operatorname{gcd}\left(f_{\alpha_{1}}, f_{\beta_{1}}\right) \operatorname{gcd}\left(f_{a_{1}} f_{a_{2}}, f_{\beta_{1}}\right) / G H,
\end{aligned}
$$

for some $E, F, G, H \in R$. It is enough to show that for any variable $x \in R$ and any positive integer $t$ if $x^{t} \mid \operatorname{gcd}\left(f_{a_{1}} f_{a_{2}}, f_{\beta_{1}}\right)$, then $x^{t} \mid E F$ and $x^{t} \mid G H$. This is equivalent to showing that if $x^{u} \mid \operatorname{gcd}\left(f_{\alpha}, f_{\beta}\right)$ and $x \mid \operatorname{gcd}\left(f_{a_{1}} f_{a_{2}}, f_{\beta_{1}}\right)$, then $x^{u} \mid \operatorname{gcd}\left(f_{a_{1}} f_{a_{2}}, f_{b_{1}} f_{b_{2}}\right) \operatorname{gcd}\left(f_{\alpha_{1}}, f_{\beta}\right)$ and $x^{u} \mid \operatorname{gcd}\left(f_{\alpha}, f_{b_{1}} f_{b_{2}}\right) \operatorname{gcd}\left(f_{\alpha_{1}}, f_{\beta_{1}}\right)$ for any positive integer $u$. But this follows immediately since $l_{1}+l_{2}>k_{1} \geq k_{2}$ and $l_{1}+l_{2}<l_{1}+l_{2}+l_{3}=$ $s=k_{1}+k_{2}$.

For claim (ii) we notice that we have the following equalities:

$$
\begin{aligned}
\operatorname{gcd}\left(f_{\alpha}, f_{\beta}\right) & =\operatorname{gcd}\left(f_{a_{1}}, f_{\beta}\right) \operatorname{gcd}\left(f_{\alpha_{1}}, f_{\beta}\right) / K \\
& =\operatorname{gcd}\left(f_{a_{1}}, f_{b_{1}}\right) \operatorname{gcd}\left(f_{a_{1}}, f_{\beta_{1}}\right) \operatorname{gcd}\left(f_{\alpha_{1}}, f_{\beta}\right) / K L, \\
& =\operatorname{gcd}\left(f_{\alpha}, f_{b_{1}}\right) \operatorname{gcd}\left(f_{\alpha}, f_{\beta_{1}}\right) / M \\
& =\operatorname{gcd}\left(f_{\alpha}, f_{b_{1}}\right) \operatorname{gcd}\left(f_{\alpha_{1}}, f_{\beta_{1}}\right) \operatorname{gcd}\left(f_{a_{1}}, f_{\beta_{1}}\right) / M N,
\end{aligned}
$$


for some $K, L, M, N \in R$. The claim follows since $l_{1}>k_{2}$.

Finally to establish the last claim we note that the assumption $l_{1} \leq k_{2}$ is equivalent to $l_{2}+l_{3} \geq k_{1}$, and thus $l_{2}+l_{3} \geq k_{1} \geq l_{1}+l_{2}$. Hence, $l_{3} \geq l_{1} \geq l_{2} \geq l_{3}$ which implies $l_{i}=s / 3$ and $2 s / 3 \leq k_{1}$. Furthermore, $s / 3 \geq k_{2} \geq s / 3$ and hence $k_{2}=s / 3$ and $k_{1}=2 s / 3$.

The next theorem is one of the main results of this section. We will use all the information we obtained about how various conditions on two sequences $\alpha, \beta \in \mathcal{I}_{s}$ affect the term $T_{\alpha, \beta}$ to obtain a bound on the relation type of $I$ as well as a description of the defining equations of the Rees algebra. Recall that the relation type of an ideal $I$ is defined to be

$$
\operatorname{rt}(I)=\min \left\{s \mid J=\bigoplus_{i=1}^{s} J_{i}\right\},
$$

where $J$ is the defining ideal of $\mathcal{R}(I)$. In other words, the relation type of $I$ is the largest degree (in the $T_{i}$ ) of any minimal generator of $J$. In particular, when $I$ is of linear type, the relation type is 1 . The relation type of an ideal has been explored in various articles, see for instance $[1,18,22,32,36]$.

Theorem 2.12. Let $R$ be a polynomial ring over a field, and let $I$ be a square-free monomial ideal generated by $n$ square-free monomials in $R$. When $n \leq 5$, then $\mathcal{R}(I)=S / J$, where $S=R\left[T_{1}, \ldots, T_{n}\right]$ and

$$
J=S J_{1}+S \cdot\left(\bigcup_{i=2}^{n-2} J_{i}\right) .
$$

In particular, $\operatorname{rt}(I) \leq n-2$.

Proof. By Theorem 2.2, it suffices to show that, given two sequences $\alpha, \beta \in \mathcal{I}_{s}$ of length $s>n-2$, then

$$
T_{\alpha, \beta} \in S J_{1}+S \cdot\left(\bigcup_{i=2}^{n-2} J_{i}\right) .
$$

Suppose that there are $l_{i}$ distinct copies of $a_{i}$ in $\alpha$ and $k_{i}$ distinct copies of $b_{i}$ in $\beta$ and write $\alpha=\left(a_{1}^{l_{1}}, \ldots, a_{m}^{l_{m}}\right)$, and $\beta=\left(b_{1}^{k_{1}}, \ldots, b_{t}^{k_{t}}\right)$. 
By Lemma 2.4 we may assume $a_{i} \neq b_{j}$ for all $i, j$. Also by Lemma 2.9, we may assume $1<m \leq 3$ and $1<t \leq 2$, since $n \leq 5$. We are now left with the following cases:

(i) Suppose that $\alpha=\left(a_{1}^{l_{1}}, a_{2}^{l_{2}}\right)$ and $\beta=\left(b_{1}^{k_{1}}, b_{2}^{k_{2}}\right)$, where $l_{1}+l_{2}=$ $k_{1}+k_{2} \geq 3$. The result follows from Lemma 2.10.

(ii) Suppose that $\alpha=\left(a_{1}^{l_{1}}, a_{2}^{l_{2}}, a_{3}^{l_{3}}\right)$ and $\beta=\left(b_{1}^{k_{1}}, b_{2}^{k_{2}}\right)$, where $l_{1}+l_{2}+$ $l_{3}=k_{1}+k_{2} \geq 4$. The result follows from Lemma 2.11 .

The following example provides a class of square-free monomial ideals generated by $n>4$ square-free monomials for which the relation type is at least $2 n-7$. Notice that when $n=5$ one has $2 n-7=n-2$. In particular, this establishes that the bound given in Theorem 2.12 is sharp. We also note that the ideals in the following example are not of fiber type.

Example 2.13. Let $R=k\left[x_{1}, x_{2}, \ldots, x_{n-2}, u_{1}, u_{2}, \ldots, u_{n-2}\right]$ be a polynomial ring over a field $k$ with $n$ a positive integer such that $n>4$. Let $I=\left(f_{1}, \ldots, f_{n}\right)$, where

$$
f_{1}=\left(\prod_{i=2}^{n-2} x_{i}\right) u_{1}, \quad f_{i}=x_{i} x_{1}\left(\prod_{j=2, j \neq i}^{n-2} u_{j}\right)
$$

for all $i=2, \ldots, n-2$,

$$
f_{n-1}=\left(\prod_{i=2}^{n-2} x_{i}\right) x_{1}, \quad \text { and } \quad f_{n}=\left(\prod_{i=2}^{n-2} u_{i}\right) u_{1} .
$$

Consider

$$
F=T_{1}^{n-4} \prod_{i=2}^{n-2} T_{i}-T_{n-1}^{n-3} T_{n}^{n-4} \quad \text { and } \quad G=u_{1} T_{1}^{n-5} \prod_{i=2}^{n-2} T_{i}-y T_{n-1}^{n-5} T_{n} .
$$

It is clear that $F$ and $G$ are in the defining ideal of the Rees algebra of $I$. Moreover, $F$ and $G$ are irreducible, and thus

$$
F \in S J_{2 n-7} \backslash S \cdot\left(\bigcup_{i=1}^{2 n-8} J_{i}\right)
$$


and

$$
G \in S J_{2 n-8} \backslash S \cdot\left(\bigcup_{i=1}^{2 n-9} J_{i}\right)
$$

Hence, the relation type of $I$ is at least $2 n-7$. Finally, we note that $G$ is not in the defining ideal of the special fiber of $I$ and therefore $I$ is not an ideal of fiber type.

The next lemma establishes conditions for when various generators of the defining ideal of the Rees algebra as in Theorem 2.12 are irredundant.

Lemma 2.14. Let $R$ be a polynomial ring over a field, and let $I$ be a square-free monomial ideal in $R$. Let $\alpha, \beta \in \mathcal{I}_{s}$ be two sequences of length $s \geq 4$, where $\mathcal{I}_{s}$ is as in Definition 2.1. Suppose that $\alpha=\left(a_{1}, \ldots, a_{s}\right), \beta=\left(b_{1}, \ldots, b_{1}, b_{2}\right)$, where $a_{i} \neq b_{j}$ for all $i, j$. Suppose that, for all $i$, there exists a variable $x_{i} \in R$ such that $x_{i} \mid f_{a_{j}}$ for all $j \neq i$ and $x_{i} \mid f_{b_{1}}, x_{i} \nmid f_{a_{i}}$, and $x_{i} \nmid f_{b_{2}}$. Furthermore, suppose that for all $i$ there exists a variable $z_{i} \in R$ such that $z_{i} \mid \operatorname{gcd}\left(f_{a_{i}}, f_{b_{2}}\right), z_{i} \nmid f_{a_{j}}$ for all $j \neq i$ and $z_{i} \nmid f_{b_{1}}$. Then

$$
T_{\alpha, \beta} \in S J_{s} \backslash S \cdot\left(\bigcup_{i=1}^{s-1} J_{i}\right)
$$

Proof. We write

$$
f_{a_{i}}=z_{i} h_{i}\left(\prod_{j=1, j \neq i}^{s} x_{j}\right)
$$

for all $i=1, \ldots, s$,

$$
f_{b_{1}}=k_{1}\left(\prod_{i=1}^{s} x_{i}\right), \quad \text { and } \quad f_{b_{2}}=k_{2}\left(\prod_{i=1}^{s} z_{i}\right)
$$

for some square-free monomials $h_{1}, \ldots, h_{s}, k_{1}, k_{2} \in R$ such that $x_{i} \nmid h_{j}$, $x_{i} \nmid k_{l}, z_{i} \nmid h_{j}$, and $z_{i} \nmid k_{l}$ for all $i, j, l$. Then we have

$$
f_{\alpha}=\prod_{i=1}^{s} x_{i}^{s-1} \prod_{i=1}^{s} z_{i} \prod_{i=1}^{s} h_{i} \quad \text { and } \quad f_{\beta}=k_{1}^{s-1} k_{2}\left(\prod_{i=1}^{s} x_{i}^{s-1} \prod_{i=1}^{s} z_{i}\right) .
$$


Let

$$
M=\frac{f_{\alpha}}{\operatorname{gcd}\left(f_{\alpha}, f_{\beta}\right)}=\frac{\prod_{i=1}^{s} h_{i}}{\operatorname{gcd}\left(\prod_{i=1}^{s} h_{i}, k_{1}^{s-1} k_{2}\right)}
$$

and

$$
N=\frac{f_{\beta}}{\operatorname{gcd}\left(f_{\alpha}, f_{\beta}\right)}=\frac{k_{1}^{s-1} k_{2}}{\operatorname{gcd}\left(\prod_{i=1}^{s} h_{i}, k_{1}^{s-1} k_{2}\right)}
$$

One can observe immediately that $x_{i} \nmid M$ and $z_{i} \nmid N$ for all $i$. Let $\alpha^{\prime}$ and $\beta^{\prime}$ be any proper subsequences of $\alpha$ and $\beta$. Then there exists either $x_{i}$ or $z_{j}$ such that

$$
x_{i} \mid \frac{f_{\alpha^{\prime}}}{\operatorname{gcd}\left(f_{\alpha^{\prime}}, f_{\beta^{\prime}}\right)} \quad \text { or } \quad z_{j} \mid \frac{f_{\alpha^{\prime}}}{\operatorname{gcd}\left(f_{\alpha^{\prime}}, f_{\beta^{\prime}}\right)}
$$

by our assumptions. Therefore,

$$
\frac{f_{\alpha^{\prime}}}{\operatorname{gcd}\left(f_{\alpha^{\prime}}, f_{\beta^{\prime}}\right)} \nmid M,
$$

and similarly,

$$
\frac{f_{\beta^{\prime}}}{\operatorname{gcd}\left(f_{\alpha^{\prime}}, f_{\beta^{\prime}}\right)} \nmid N .
$$

This shows that

$$
T_{\alpha, \beta} \in S J_{s} \backslash S \cdot\left(\bigcup_{i=1}^{s-1} J_{i}\right)
$$

The following theorem establishes precisely the defining equations for the Rees algebra of a square-free monomial ideal with up to five generators.

Theorem 2.15. Let $R$ be a polynomial ring over a field, and let $I$ be a square-free monomial ideal generated by $n$ square-free monomials in $R$. Suppose that $n \leq 5$. Suppose that there does not exist a pair of sequences $\alpha, \beta \in \mathcal{I}_{s}$ of length $s \geq 2$ as in Definition 2.1, such that the conditions of Lemma 2.14 are satisfied. Then $I$ is an ideal of linear type. 
Proof. Consider a pair of sequences $\alpha, \beta$ as in Definition 2.1 of length $s \geq 2$ such that $\alpha=\left(a_{1}, \ldots, a_{s}\right), \beta=\left(b_{1}, \ldots, b_{1}, b_{2}\right)$, where $a_{i} \neq b_{j}$ for all $i, j$. Suppose that one of the following conditions is not satisfied: For all $i$, there exists a variable $x_{i} \in R$ such that $x_{i} \mid f_{a_{j}}$ for all $j \neq i$, $x_{i} \mid f_{b_{1}}, x_{i} \nmid f_{a_{i}}$, and $x_{i} \nmid f_{b_{2}}$, and for all $i$ there exists a variable $z_{i} \in R$ such that $z_{i} \mid \operatorname{gcd}\left(f_{a_{i}}, f_{b_{2}}\right), z_{i} \nmid f_{a_{j}}$ for all $j \neq i$ and $z_{i} \nmid f_{b_{1}}$.

We will show that

$$
T_{\alpha, \beta} \in S J_{1}+S \cdot\left(\bigcup_{i=1}^{s-1} J_{i}\right)
$$

and by induction $T_{\alpha, \beta} \in S J_{1}$. By Theorem 2.12 and Lemmas 2.4 and 2.9 it is enough to consider sequences of length 2 or 3, i.e., either $\alpha=\left(a_{1}, a_{2}\right)$ and $\beta=\left(b_{1}, b_{2}\right)$ or $\alpha=\left(a_{1}, a_{2}, a_{3}\right)$ and $\beta=\left(b_{1}, b_{1}, b_{2}\right)$. The proof for the first case can be treated as a special case of the second and thus we will only consider the case with $\alpha=\left(a_{1}, a_{2}, a_{3}\right)$ and $\beta=\left(b_{1}, b_{1}, b_{2}\right)$. Without lost of generality, we will show that if there does not exist a variable $x \in R$ such that $x \mid \operatorname{gcd}\left(f_{a_{2}}, f_{a_{3}}\right)$, $x \mid f_{b_{1}}, x \nmid f_{a_{1}}$, and $x \nmid f_{b_{2}}$ then

$$
\begin{aligned}
\operatorname{gcd}\left(f_{a_{1}} f_{a_{2}} f_{a_{3}}, f_{b_{1}}^{2} f_{b_{2}}\right) & =\operatorname{gcd}\left(f_{a_{1}}, f_{b_{1}}\right) \operatorname{gcd}\left(f_{\alpha}, f_{b_{1}} f_{b_{2}}\right) / A \\
& =\operatorname{gcd}\left(f_{a_{1}}, f_{\beta}\right) \operatorname{gcd}\left(f_{a_{2}} f_{a_{3}}, f_{b_{1}} f_{b_{2}}\right) / B,
\end{aligned}
$$

for some $A, B \in R$.

Similarly, if there does not exist a variable $z \in R$ such that

$$
\begin{aligned}
& z \mid \operatorname{gcd}\left(f_{a_{1}}, f_{b_{2}}\right), \quad z \nmid f_{a_{j}} \text { for } j=2,3 \text { and } z \nmid f_{b_{1}} \text { then } \\
& \operatorname{gcd}\left(f_{a_{1}} f_{a_{2}} f_{a_{3}}, f_{b_{1}}^{2} f_{b_{2}}\right)=\operatorname{gcd}\left(f_{a_{1}}, f_{b_{1}}\right) \operatorname{gcd}\left(f_{a_{2}} f_{a_{3}}, f_{\beta}\right) / C \\
&=\operatorname{gcd}\left(f_{\alpha}, f_{b_{1}}\right) \operatorname{gcd}\left(f_{a_{2}} f_{a_{3}}, f_{b_{1}} f_{b_{2}}\right) / D,
\end{aligned}
$$

for some $C, D \in R$. Hence, we may apply Lemma 2.7 to obtain the result.

For the first part, we notice that

$$
\begin{aligned}
& \operatorname{gcd}\left(f_{a_{1}} f_{a_{2}} f_{a_{3}}, f_{b_{1}}^{2} f_{b_{2}}\right) \\
& =\operatorname{gcd}\left(f_{\alpha}, f_{b_{1}}\right) \operatorname{gcd}\left(f_{\alpha}, f_{b_{1}} f_{b_{2}}\right) / E \\
& =\operatorname{gcd}\left(f_{a_{1}}, f_{b_{1}}\right) \operatorname{gcd}\left(f_{a_{2}} f_{a_{3}}, f_{b_{1}}\right) \operatorname{gcd}\left(f_{\alpha}, f_{b_{1}} f_{b_{2}}\right) / E F, \\
& \operatorname{gcd}\left(f_{a_{1}} f_{a_{2}} f_{a_{3}}, f_{b_{1}}^{2} f_{b_{2}}\right)
\end{aligned}
$$




$$
\begin{aligned}
& =\operatorname{gcd}\left(f_{a_{1}}, f_{\beta}\right) \operatorname{gcd}\left(f_{a_{2}} f_{a_{3}}, f_{\beta}\right) / G \\
& =\operatorname{gcd}\left(f_{a_{1}}, f_{\beta}\right) \operatorname{gcd}\left(f_{a_{2}} f_{a_{3}}, f_{b_{1}}\right) \operatorname{gcd}\left(f_{a_{2}} f_{a_{3}}, f_{b_{1}} f_{b_{2}}\right) / G H,
\end{aligned}
$$

for some $E, F, G, H \in R$. It is enough to show for any variable $x \in R$ if $x \mid \operatorname{gcd}\left(f_{a_{2}} f_{a_{3}}, f_{b_{1}}\right)$, then $x \mid E F$ and $x \mid G H$. This is equivalent to showing that for any integer $u$ if $x^{u} \mid \operatorname{gcd}\left(f_{\alpha}, f_{\beta}\right)$ then $x^{u} \mid \operatorname{gcd}\left(f_{a_{1}}, f_{b_{1}}\right) \operatorname{gcd}\left(f_{\alpha}, f_{b_{1}} f_{b_{2}}\right)$ and $x^{u} \mid \operatorname{gcd}\left(f_{a_{1}}, f_{\beta}\right) \operatorname{gcd}\left(f_{a_{2}} f_{a_{3}}, f_{b_{1}} f_{b_{2}}\right)$. This is straightforward to verify and the only case that is not trivial is when $x \nmid f_{a_{1}}$ and $x \mid \operatorname{gcd}\left(f_{a_{2}}, f_{a_{3}}\right)$. Then $x \mid f_{b_{2}}$ by assumption. Hence, $x^{2} \mid \operatorname{gcd}\left(f_{\alpha}, f_{b_{1}} f_{b_{2}}\right)$ and $x^{2} \mid \operatorname{gcd}\left(f_{a_{2}} f_{a_{3}}, f_{b_{1}} f_{b_{2}}\right)$.

We can use a similar argument for the second part, and the only case that is not trivial is when $x \mid \operatorname{gcd}\left(f_{a_{1}}, f_{b_{1}} f_{b_{2}}\right)$ and $x \nmid f_{b_{1}}$. Then $x \mid f_{b_{2}}$ and $x \mid f_{a_{2}}$ or $x \mid f_{a_{3}}$ by assumption. Hence, $x^{2} \nmid \operatorname{gcd}\left(f_{\alpha}, f_{\beta}\right)$, $x \mid \operatorname{gcd}\left(f_{a_{2}} f_{a_{3}}, f_{\beta}\right)$ and $x \mid \operatorname{gcd}\left(f_{a_{2}} f_{a_{3}}, f_{b_{1}} f_{b_{2}}\right)$.

We now introduce a construction that is also known as the line graph of the hypergraph corresponding to a monomial ideal.

Construction 2.16. Let $R$ be a polynomial ring over a field, and let $I$ be a monomial ideal in $R$. Let $f_{1}, \ldots, f_{n}$ be a minimal monomial generating set of $I$. We construct the following graph $\widetilde{G}(I)$ For each $f_{i}$ we associate a vertex $y_{i}$ to it. The edges of this graph are $\left\{y_{i}, y_{j}\right\}$, where $\operatorname{gcd}\left(f_{i}, f_{j}\right) \neq 1$. We call the graph $\widetilde{G}(I)$ the line graph of $I$.

The purpose of introducing the line graph of the hypergraph corresponding to a monomial ideal is to utilize the graph structure in order to determine combinatorial conditions that determine the defining equations of the Rees algebra. We first observe the following.

Remark 2.17. With the same assumptions as in Lemma 2.14 the sequences $\alpha, \beta$ correspond to an even closed walk in $\widetilde{G}(I)$. Indeed, the following

$$
\begin{gathered}
\left\{y_{b_{1}},\left\{y_{b_{1}}, y_{a_{1}}\right\}, y_{a_{1}},\left\{y_{a_{1}}, y_{b_{1}}\right\}, y_{b_{1}},\left\{y_{b_{1}}, y_{a_{2}}\right\}, y_{a_{2}},\left\{y_{a_{2}}, y_{b_{1}}\right\}, y_{b_{1}}, \ldots,\right. \\
\left.y_{b_{1}},\left\{y_{b_{1}}, y_{a_{s-1}}\right\}, y_{a_{s-1}},\left\{y_{a_{s-1}}, y_{b_{2}}\right\}, y_{b_{2}},\left\{y_{b_{2}}, y_{a_{s}}\right\}, y_{a_{s}},\left\{y_{a_{s}}, y_{b_{1}}\right\}, y_{b_{1}}\right\}
\end{gathered}
$$


is an even closed walk and

$$
T_{\alpha, \beta} \in S J_{s} \backslash S \cdot\left(\bigcup_{i=1}^{s-1} J_{i}\right) .
$$

We now introduce the notion of a subgraph induced by two sequences.

Definition 2.18. Let $R$ be a polynomial ring over a field, and let $I$ be a monomial ideal in $R$. Let $\alpha, \beta \in \mathcal{I}_{s}$ be two sequences of length $s \geq 2$, where $\mathcal{I}_{s}$ is as in Definition 2.1. Let $\alpha=\left(a_{1}^{l_{1}}, a_{2}^{l_{2}}, \ldots, a_{m}^{l_{m}}\right)$ and $\beta=$ $\left(b_{1}^{r_{1}}, \ldots, b_{t}^{r_{t}}\right)$, where $l_{i}$ and $r_{i}$ are the number of distinct copies of $a_{i}$ and $b_{i}$, respectively. Let $\alpha_{1}=\left(a_{1}, \ldots, a_{m}\right)$ and $\beta_{1}=\left(b_{i_{1}}, \ldots, b_{i_{r}}\right)$, where $\left\{b_{i_{1}}, \ldots, b_{i_{r}}\right\}=\left\{b_{1}, \ldots, b_{t}\right\} \backslash\left\{a_{1}, \ldots, a_{m}\right\}$. The last condition ensures that $a_{i} \neq b_{i_{k}}$ for all $i, i_{k}$. Consider $K=\left(f_{a_{1}}, \ldots, f_{a_{m}}, f_{b_{i_{1}}}, \ldots, f_{b_{i_{r}}}\right)$ and the line graph $\widetilde{G}(K)$ of $K$. Notice that $K \subset I$ and that $\widetilde{G}(K)$ is a subgraph of $\widetilde{G}(I)$, since $a_{i} \neq b_{i_{k}}$ for all $i, i_{k}$. We call $\widetilde{G}(K)$ the graph induced by $\alpha$ and $\beta$.

Remark 2.19. Let $R$ be a polynomial ring over a field, and let $I$ be a monomial ideal in $R$. Let $\alpha, \beta \in \mathcal{I}_{s}$ be two sequences of length $s \geq 2$, where $\mathcal{I}_{s}$ is as in Definition 2.1. Let $G$ be the graph induced by $\alpha$ and $\beta$. If $G$ is a disconnected graph, then

$$
T_{\alpha, \beta} \in S J_{1}+S \cdot\left(\bigcup_{i=2}^{s-1} J_{i}\right),
$$

by Proposition 2.8 .

The following theorem is an extension of Theorem 2.12. We use the line graph to give a description for the defining equations of the Rees algebra.

Theorem 2.20. Let $R$ be a polynomial ring over a field, and let I be a square-free monomial ideal generated by $n$ square-free monomials in $R$. Suppose that the line graph of $I$ is the disjoint union of graphs with at most 5 vertices. Let $\mathcal{R}(I)=S / J$, where $S=R\left[T_{1}, \ldots, T_{n}\right]$. Then $J=S J_{1}+S \cdot\left(J_{2} \cup J_{3}\right)$ and in particular, $\operatorname{rt}(I) \leq 3$. Furthermore, if 
$T_{\alpha, \beta} \in S \cdot\left(J_{2} \cup J_{3}\right) \backslash S J_{1}$, then the subgraph induced by $\alpha, \beta$ is an even closed walk of $\widetilde{G}(I)$.

Proof. By Remark 2.19, we may assume that $\widetilde{G}(I)$ is connected. Then the first part follows immediately by Theorem 2.12. The last part follows from Theorem 2.15, Lemma 2.14 and Remark 2.17.

The next example illustrates the result of Theorem 2.20.

Example 2.21. Let $R=k\left[x_{1}, \ldots, x_{7}\right]$ be a polynomial ring over a field $k$. Let $I=\left(f_{1}, \ldots, f_{5}\right)$, where $f_{1}=x_{1} x_{2} x_{3}, f_{2}=x_{1} x_{2} x_{4} x_{7}$, $f_{3}=x_{2} x_{3} x_{6}, f_{4}=x_{4} x_{5} x_{6}$, and $f_{5}=x_{1} x_{3} x_{5}$. Let $\mathcal{R}(I)=S / J$, where $S=R\left[T_{1}, \ldots, T_{5}\right]$. Let $\alpha=(1,1,4)$ and $\beta=(2,3,5)$. The subgraph induced by $\alpha, \beta$ is the even closed walk

$$
\left\{y_{1},\left\{y_{1}, y_{2}\right\}, y_{2},\left\{y_{1}, y_{2}\right\}, y_{1},\left\{y_{1}, y_{3}\right\}, y_{3},\left\{y_{3}, y_{4}\right\}, y_{4},\left\{y_{4}, y_{5}\right\}, y_{5},\left\{y_{1}, y_{5}\right\}\right\}
$$

of the line graph of $I$ shown below

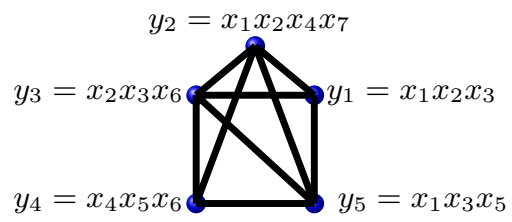

Then by Theorem 2.20 we have that $T_{\alpha, \beta}=T_{2} T_{3} T_{5}-x_{7} T_{1}^{2} T_{4} \in$ $S J_{3} \backslash\left(S J_{1}+S J_{2}\right)$, since it is irreducible.

The following example comes from [35, Example 3.1]. Villarreal used this example to show that his methods do not extend in the case of square-free monomial ideals generated in degree higher than 2 . In light of Theorem 2.20 we now give an explicit description of the defining equations of the Rees algebra for this example.

Example 2.22. Let $R=k\left[x_{1}, \ldots, x_{7}\right]$, where $k$ is a field, and let $I$ be an ideal of $R$ generated by $f_{1}=x_{1} x_{2} x_{3}, f_{2}=x_{2} x_{4} x_{5}, f_{3}=x_{5} x_{6} x_{7}$, $f_{4}=x_{3} x_{6} x_{7}$. Then the defining ideal of $\mathcal{R}(I)$ is minimally generated 
by the binomials

$$
\begin{aligned}
x_{3} T_{3}-x_{5} T_{4}, x_{6} x_{7} T_{1}-x_{1} x_{2} T_{4}, x_{6} x_{7} T_{2} & -x_{2} x_{4} T_{3}, x_{4} x_{5} T_{1} \\
& -x_{1} x_{3} T_{2}, x_{4} T_{1} T_{3}-x_{1} T_{2} T_{4} .
\end{aligned}
$$

Notice that the only binomial that is not linear is $x_{4} T_{1} T_{3}-x_{1} T_{2} T_{4}$ and it comes from the unique even cycle of $\widetilde{G}(I)$, which in this case is the graph of a square.

Before concluding this section, we turn our attention to toric ideals. Recall that a toric ideal is the ideal of relations of a monomial subring of a polynomial ring. Let $\mathcal{A}=\left\{a_{1}, \ldots, a_{n}\right\} \in \mathbb{N}^{c} \backslash\{0\}$, and let $A$ be a matrix with columns $a_{i}$ and suppose that $A$ has rank $c$. Let $k$ be a field, and let $S=k\left[\overline{T_{1}}, \ldots, T_{n}\right]$ be a polynomial ring over $k$. Let $\phi: S \rightarrow k\left[t_{1}, \ldots, t_{c}\right]$ be a map defined by $\phi\left(x_{i}\right)=\underline{t} \underline{a_{i}}$. Then $\operatorname{ker} \phi$ is a prime ideal and it is called the toric ideal associated to $\mathcal{A}$. In particular, when $I$ is generated by square-free monomials of the same degree, then the defining ideal of the special fiber $\operatorname{ring} \mathcal{F}(I)=\mathcal{R}(I) \otimes k$ is the toric ideal associated to $I$. Notice that in this case $\mathcal{F}(I)=k\left[f_{1}, \ldots, f_{n}\right]$, where $f_{1}, \ldots, f_{n}$ is a minimal monomial generating set of $I$. In light of Theorem 2.20 we can give a concrete description of such toric ideals.

Corollary 2.23. Let $R$ be a polynomial ring over a field $k$, let $f_{1}, \ldots, f_{n}$ be square-free monomials of the same degree, and let $I=$ $\left(f_{1}, \ldots, f_{n}\right)$. Suppose that the line graph of $I$ is the disjoint union of graphs with at most 5 vertices. Let $\mathcal{F}(I)=k\left[f_{1}, \ldots, f_{n}\right]$ be the monomial subring of $R$ generated by $f_{1}, \ldots, f_{n}$. Then $\mathcal{F}(I) \simeq k\left[T_{1}, \ldots, T_{n}\right] / J$ and the toric ideal $J$ corresponding to $I$ is generated by

$$
\begin{aligned}
\left\{T_{\alpha}-T_{\beta} \mid \alpha, \beta \in\right. & \mathcal{I}_{s}, \text { with } s=2,3 \text {, and } f_{\alpha}=f_{\beta}, \\
& \text { where } \alpha, \beta \text { induce an even closed walk in } \widetilde{G}(I)\} .
\end{aligned}
$$

3. Square-free monomial ideals of linear type. In this section we turn our attention to ideals of linear type. Villarreal showed that when $I$ is the edge ideal of a graph then $I$ is of linear type if and only if the graph of $I$ is the disjoint union of graphs of trees and graphs that have a unique odd cycle, [35, Corollary 3.2]. Inspired by this result, we use the line graph associated to any monomial ideal as in 
Construction 2.16 in order to obtain similar results as Villarreal for any square-free monomial ideal without restrictions on the degrees of the generators. A natural first class to consider is ideals with line graph the graph of a forest.

Proposition 3.1. Let $R$ be a polynomial ring over a field and let $I$ be a square-free monomial ideal in $R$. We further assume that the line graph of $I$ is the graph of a forest. Then $I$ is an ideal of linear type.

Proof. Let $f_{1}, \ldots, f_{n}$ be a minimal monomial generating set for $I$. We show that for all sequences $\alpha, \beta \in \mathcal{I}_{s}$ of length $s \geq 2$, where $\mathcal{I}_{s}$ is as in Definition 2.1, we have

$$
T_{\alpha, \beta} \in S J_{1}+S \cdot\left(\bigcup_{i=2}^{s-1} J_{i}\right)
$$

and by induction it follows that $T_{\alpha, \beta} \in S J_{1}$.

Since $\widetilde{G}(I)$ is the graph of a forest, then every subgraph of $\widetilde{G}(I)$ is also a forest. By Remark 2.19, we may assume that $\widetilde{G}(I)$ is connected, i.e., it is the graph of a tree. Notice that for every graph of a tree there exists a vertex that is only connected to one other vertex.

Suppose that $s \geq 2$. Let $\alpha=\left(a_{1}^{l_{1}}, a_{2}^{l_{2}}, \ldots, a_{m}^{l_{m}}\right)$ and $\beta=$ $\left(b_{1}^{r_{1}}, \ldots, b_{t}^{r_{t}}\right)$, where $l_{i}$ and $r_{i}$ are the number of distinct copies of $a_{i}$ and $b_{i}$, respectively. Notice that, if $a_{i}=b_{j}$ for some $i$ and some $j$, then the result follows by Lemma 2.4 and the induction hypothesis. Hence, we may assume that $a_{i} \neq b_{j}$ for all $i, j$. Let $\alpha_{1}=\left(a_{1}, \ldots, a_{m}\right)$ and $\beta_{1}=\left(b_{1}, \ldots, b_{t}\right)$. Then the graph $G^{\prime}$ induced by $\alpha_{1}, \beta_{1}$ is a subgraph of $\widetilde{G}(I)$, by Definition 2.18. Hence $G^{\prime}$ is the graph of a forest. If $G^{\prime}$ is disconnected, then the result follows by Remark 2.19 and the induction hypothesis. Therefore, we may assume that $G^{\prime}$ is connected and hence the graph of a tree. Thus, without loss of generality, we may assume that $y_{a_{1}}$ is only connected to $y_{b_{1}}$.

If $l_{1} \geq r_{1}$, then $\operatorname{gcd}\left(f_{a_{1}}, f_{b_{2}}^{r_{2}} \cdots f_{b_{t}}^{r_{t}}\right)=1$, and hence

$$
T_{\alpha, \beta} \in S J_{1}+S \cdot\left(\bigcup_{i=2}^{s-1} J_{i}\right)
$$


by Proposition 2.8. So we may assume that $l_{1}<r_{1}$. Then we claim that

(a) $\operatorname{gcd}\left(f_{\alpha}, f_{\beta}\right)=\operatorname{gcd}\left(f_{a_{1}}^{l_{1}}, f_{b_{1}}^{l_{1}}\right) \operatorname{gcd}\left(f_{a_{2}}^{l_{2}} \cdots f_{a_{m}}^{l_{m}}, f_{\beta}\right)$,

(b) $\operatorname{gcd}\left(f_{\alpha}, f_{\beta}\right)=\operatorname{gcd}\left(f_{\alpha}, f_{b_{1}}^{l_{1}}\right) \operatorname{gcd}\left(f_{a_{2}}^{l_{2}} \cdots f_{a_{m}}^{l_{k}}, f_{b_{1}}^{r_{1}-l_{1}} f_{b_{2}}^{r_{2}} \cdots f_{b_{t}}^{r_{t}}\right) / A$,

for some $A \in R$. Notice that, by the claim and Lemma 2.7, it follows that

$$
T_{\alpha, \beta} \in S J_{1}+S \cdot\left(\bigcup_{i=2}^{s-1} J_{i}\right) .
$$

Therefore, it remains to prove the claim.

For part (a), notice that

$$
\begin{aligned}
\operatorname{gcd}\left(f_{\alpha}, f_{\beta}\right) & =\operatorname{gcd}\left(f_{a_{1}}^{l_{1}}, f_{\beta}\right) \operatorname{gcd}\left(f_{a_{2}}^{l_{2}} \cdots f_{a_{m}}^{l_{m}}, f_{\beta}\right) \\
& =\left(f_{a_{1}}^{l_{1}}, f_{b_{1}}^{l_{1}}\right) \operatorname{gcd}\left(f_{a_{2}}^{l_{2}} \cdots f_{a_{m}}^{l_{m}}, f_{\beta}\right),
\end{aligned}
$$

where the equalities follow from the fact that

$$
\begin{gathered}
\operatorname{gcd}\left(f_{a_{1}}, f_{a_{i}}\right)=\operatorname{gcd}\left(f_{a_{1}}, f_{b_{j}}\right)=1 \\
\text { for any } i>1, j>1,
\end{gathered}
$$

and Remark 2.6 (b).

For part (b), notice that

$\operatorname{gcd}\left(f_{\alpha}, f_{\beta}\right)$

$=\operatorname{gcd}\left(f_{\alpha}, f_{b_{1}}^{l_{1}}\right) \operatorname{gcd}\left(f_{\alpha}, f_{b_{1}}^{r_{1}-l_{1}} f_{b_{2}}^{r_{2}} \cdots f_{b_{t}}^{r_{t}}\right) / B$

$=\operatorname{gcd}\left(f_{\alpha}, f_{b_{1}}^{l_{1}}\right) \operatorname{gcd}\left(f_{a_{1}}^{l_{1}}, f_{b_{1}}^{r_{1}-l_{1}} f_{b_{2}}^{r_{2}} \cdots f_{b_{t}}^{r_{t}}\right) \operatorname{gcd}\left(f_{a_{2}}^{l_{2}} \cdots f_{a_{m}}^{l_{m}}, f_{b_{1}}^{r_{1}-l_{1}} f_{b_{2}}^{r_{2}} \cdots f_{b_{t}}^{r_{t}}\right) / B C$

$=\operatorname{gcd}\left(f_{\alpha}, f_{b_{1}}^{l_{1}}\right) \operatorname{gcd}\left(f_{a_{1}}^{l_{1}}, f_{b_{1}}^{r_{1}-l_{1}}\right) \operatorname{gcd}\left(f_{a_{2}}^{l_{2}} \cdots f_{a_{m}}^{l_{m}}, f_{b_{2}}^{r_{2}} \cdots f_{b_{t}}^{r_{t}}\right) / B C$,

for some $B, C \in R$, since $\operatorname{gcd}\left(f_{a_{1}}^{l_{1}}, f_{b_{2}}^{r_{2}} \cdots f_{b_{t}}^{r_{t}}\right)=1$. It is enough to show that $\operatorname{gcd}\left(f_{a_{1}}^{l_{1}}, f_{b_{1}}^{r_{1}-l_{1}}\right) \mid B C$, i.e., for any variable $x \in R$ and any integer $v$ if $x^{v} \mid \operatorname{gcd}\left(f_{a_{1}}^{l_{1}}, f_{b_{1}}^{r_{1}-l_{1}}\right)$, then $x^{v} \mid B C$. This is equivalent to showing that for any variable $x \in R$ if $x \mid \operatorname{gcd}\left(f_{a_{1}}^{l_{1}}, f_{b_{1}}^{r_{1}-l_{1}}\right)$ and $x^{u} \mid \operatorname{gcd}\left(f_{\alpha}, f_{\beta}\right)$ for some integer $u$, then $x^{u} \mid \operatorname{gcd}\left(f_{\alpha}, f_{b_{1}}^{l_{1}}\right) \operatorname{gcd}\left(f_{a_{2}}^{l_{2}} \cdots f_{a_{m}}^{l_{m}}, f_{b_{1}}^{r_{1}-l_{1}} f_{b_{2}}^{r_{2}} \cdots f_{b_{t}}^{r_{t}}\right)$. If $x \mid \operatorname{gcd}\left(f_{a_{1}}^{l_{1}}, f_{b_{1}}^{r_{1}-l_{1}}\right)$ and $x^{u} \mid \operatorname{gcd}\left(f_{\alpha}, f_{\beta}\right)$, then $u \leq l_{1}$ by the fact that $y_{a_{1}}$ is only connected to $y_{b_{1}}$ and $l_{1}<r_{1}$. Therefore, $x^{u} \mid \operatorname{gcd}\left(f_{\alpha}, f_{b_{1}}^{l_{1}}\right)$. 
The following lemma allows us to handle the induction step in the case of odd cycles, in order to prove that when the line graph of a square-free monomial ideal is the graph of an odd cycle, then the ideal is of linear type.

Lemma 3.2. Let $R$ be a polynomial ring over a field, and let $I$ be a square-free monomial ideal in $R$. Let $\alpha, \beta \in \mathcal{I}_{s}$ be two sequences of length $s \geq 4$, where $\mathcal{I}_{s}$ is as in Definition 2.1. Let $\alpha=\left(a_{1}^{l_{1}}, a_{2}^{l_{2}}, \ldots, a_{m}^{l_{m}}\right)$ and $\beta=\left(b_{1}^{r_{1}}, \ldots, b_{t}^{r_{t}}\right)$, where $l_{i}$ and $r_{i}$ are the number of distinct copies of $a_{i}$ and $b_{i}$, respectively. Suppose that $l_{1}<r_{1}, l_{2}<r_{2}$, and that the graph $\widetilde{G}(I)$ is the graph of an odd cycle of length at least 5 . We further assume that $y_{a_{1}}$ is connected to $y_{b_{1}}$ and $y_{a_{2}}$ only, and $y_{a_{2}}$ is connected to $y_{a_{1}}$ and $y_{b_{2}}$ only. Then

$$
T_{\alpha, \beta} \in S J_{1}+S \cdot\left(\bigcup_{i=2}^{s-1} J_{i}\right) .
$$

Proof. Let $\alpha^{\prime}=\left(a_{3}^{l_{3}}, \ldots, a_{m}^{l_{m}}\right)$ and $\beta^{\prime}=\left(b_{1}^{r_{1}-l_{1}}, b_{2}^{r_{2}-l_{2}}, b_{3}^{r_{3}}, \ldots, b_{t}^{r_{t}}\right)$. Using Remarks 2.3 and 2.6 we have that

$$
\begin{aligned}
\operatorname{gcd}\left(f_{\alpha}, f_{\beta}\right) & =\operatorname{gcd}\left(f_{a_{1}}^{l_{1}} f_{a_{2}}^{l_{2}}, f_{\beta}\right) \operatorname{gcd}\left(f_{\alpha^{\prime}}, f_{\beta}\right) \\
& =\operatorname{gcd}\left(f_{a_{1}}^{l_{1}} f_{a_{2}}^{l_{2}}, f_{b_{1}}^{l_{1}} f_{b_{2}}^{l_{2}}\right) \operatorname{gcd}\left(f_{\alpha^{\prime}}, f_{\beta}\right),
\end{aligned}
$$

since $l_{1}<r_{1}$ and $l_{2}<r_{2}$.

We claim that $\operatorname{gcd}\left(f_{\alpha}, f_{\beta}\right)=\operatorname{gcd}\left(f_{\alpha}, f_{b_{1}}^{l_{1}} f_{b_{2}}^{l_{2}}\right)\left(f_{\alpha^{\prime}}, f_{\beta^{\prime}}\right) / C$, for some $C \in R$. Then the result will follow by Lemma 2.7. Notice that

$$
\begin{aligned}
\operatorname{gcd}\left(f_{\alpha}, f_{\beta}\right) & =\operatorname{gcd}\left(f_{\alpha}, f_{b_{1}}^{l_{1}} f_{b_{2}}^{l_{2}}\right) \operatorname{gcd}\left(f_{\alpha}, f_{\beta^{\prime}}\right) / D \\
& =\operatorname{gcd}\left(f_{\alpha}, f_{b_{1}}^{l_{1}} f_{b_{2}}^{l_{2}}\right) \operatorname{gcd}\left(f_{\alpha^{\prime}}, f_{\beta^{\prime}}\right) \operatorname{gcd}\left(f_{a_{1}}^{l_{1}} f_{a_{2}}^{l_{2}}, f_{\beta^{\prime}}\right) / D E \\
& =\operatorname{gcd}\left(f_{\alpha}, f_{b_{1}}^{l_{1}} f_{b_{2}}^{l_{2}}\right) \operatorname{gcd}\left(f_{\alpha^{\prime}}, f_{\beta^{\prime}}\right) \operatorname{gcd}\left(f_{a_{1}}^{l_{1}} f_{a_{2}}^{l_{2}}, f_{b_{1}}^{r_{1}-l_{1}} f_{b_{2}}^{r_{2}-l_{2}}\right) / D E,
\end{aligned}
$$

where $D, E \in R$ and the third equality follows from the fact that $y_{a_{1}}$ and $y_{a_{2}}$ are only connected to $y_{b_{1}}$ and $y_{b_{2}}$, respectively. It is enough to show that for any variable $x \in R$ and any integer $v$ if $x^{v} \mid \operatorname{gcd}\left(f_{a_{1}}^{l_{1}} f_{a_{2}}^{l_{2}}, f_{b_{1}}^{r_{1}-l_{1}} f_{b_{2}}^{r_{2}-l_{2}}\right)$, then $x^{v} \mid D E$. This is equivalent to showing that if $x \mid \operatorname{gcd}\left(f_{a_{1}}^{l_{1}} f_{a_{2}}^{l_{2}}, f_{b_{1}}^{r_{1}-l_{1}} f_{b_{2}}^{r_{2}-l_{2}}\right)$ and $x^{u} \mid \operatorname{gcd}\left(f_{\alpha}, f_{\beta}\right)$, then $x^{u} \mid \operatorname{gcd}\left(f_{\alpha}, f_{b_{1}}^{l_{1}} f_{b_{2}}^{l_{2}}\right) \operatorname{gcd}\left(f_{\alpha^{\prime}}, f_{\beta^{\prime}}\right)$. If $x \mid \operatorname{gcd}\left(f_{a_{1}}^{l_{1}} f_{a_{2}}^{l_{2}}, f_{b_{1}}^{r_{1}-l_{1}} f_{b_{2}}^{r_{2}-l_{2}}\right)$, then $x$ cannot divide both $f_{a_{1}}$ and $f_{a_{2}}$, since otherwise $\left\{y_{a_{1}}, y_{a_{2}}, y_{b_{1}}\right\}$ or 
$\left\{y_{a_{1}}, y_{a_{2}}, y_{b_{2}}\right\}$ will form a 3 -cycle in $\widetilde{G}(I)$. Similarly, $x$ cannot divide both $f_{b_{1}}$ and $f_{b_{2}}$. Therefore, without loss of generality we may assume $x \mid f_{a_{1}}$ and $x \mid f_{b_{1}}$. Since $l_{1}<r_{1}$ and $y_{a_{1}}$ is not connected to $y_{a_{i}}$ for all $i>2$, we obtain that if $x^{u} \mid \operatorname{gcd}\left(f_{\alpha}, f_{\beta}\right)$, then $u \leq l_{1}$. Therefore, $x^{u} \mid \operatorname{gcd}\left(f_{\alpha}, f_{b_{1}}^{l_{1}} f_{b_{2}}^{l_{2}}\right)$.

In the next proposition we are able to handle the case where each of the connected components of the line graph has a unique odd cycle.

Proposition 3.3. Let $R$ be a polynomial ring over a field, and let $I$ be a square-free monomial ideal in $R$. We further assume that line graph $\widetilde{G}(I)$ of I is the disjoint union of graphs with a unique odd cycle. Then $I$ is an ideal of linear type.

Proof. By Remark 2.19, it suffices to consider the connected components of $\widetilde{G}(I)$. Hence, we may assume that $\widetilde{G}(I)$ is connected and it has a unique odd cycle. We show that for all sequences $\alpha, \beta \in \mathcal{I}_{s}$ of length $s \geq 2$, where $\mathcal{I}_{s}$ is as in Definition 2.1, we have

$$
T_{\alpha, \beta} \in S J_{1}+S \cdot\left(\bigcup_{i=2}^{s-1} J_{i}\right) .
$$

By induction it will follow that $T_{\alpha, \beta} \in S J_{1}$.

Let $\alpha=\left(a_{1}^{l_{1}}, a_{2}^{l_{2}}, \ldots, a_{m}^{l_{m}}\right)$ and $\beta=\left(b_{1}^{r_{1}}, \ldots, b_{t}^{r_{t}}\right)$, where $l_{i}$ and $r_{i}$ are the number of distinct copies of $a_{i}$ and $b_{i}$, respectively. Using Lemma 2.4 we may assume without loss of generality that $a_{i} \neq b_{j}$ for all $i$ and $j$. Let $\alpha_{1}=\left(a_{1}, \ldots, a_{m}\right)$ and $\beta_{1}=\left(b_{1}, \ldots, b_{t}\right)$. Then the graph $G^{\prime}$ induced by $\alpha_{1}$ and $\beta_{1}$ is a subgraph of $\widetilde{G}(I)$, by Definition 2.18. By Remark 2.19, we may assume that $G^{\prime}$ is a connected graph. Notice that if there exists a vertex $y_{i}$ that is only connected to one of the $y_{j}$ then

$$
T_{\alpha, \beta} \in S J_{1}+S \cdot\left(\bigcup_{i=2}^{s-1} J_{i}\right)
$$

by the proof of Proposition 3.1. Hence, we may assume that $G^{\prime}$ is the graph of an odd cycle. Therefore, every vertex is only connected to two other vertices. 
Let $l=m+t$. Since $G^{\prime}$ is the graph of an odd cycle then $l$ is odd and hence $m \neq t$. We proceed by induction on $l$. If $l=3$, then $\widetilde{G}(I)$ is the graph of a triangle and the result follows from Lemmas 2.4 and 2.9. Suppose that $l \geq 5$. Using Lemma 2.9, we may assume that $m \geq 2$ and $t \geq 2$. Notice that $y_{a_{1}}$ is connected to two other vertices. Without loss of generality, we may assume that we have the following possible cases

(i) $y_{a_{1}}$ is connected only to $y_{a_{2}}$ and $y_{a_{3}}$,

(ii) $y_{a_{1}}$ is connected only to $y_{a_{2}}$ and $y_{b_{1}}$,

(iii) $y_{a_{1}}$ is connected only to $y_{b_{1}}$ and $y_{b_{2}}$.

For case (i), we note that since $y_{a_{1}}$ is connected only to $y_{a_{2}}$ and $y_{a_{3}}$, then using $k=l_{1}$ in Proposition 2.8 and induction we obtain the result.

For case (ii), suppose that $y_{a_{1}}$ is connected only to $y_{a_{2}}$ and $y_{b_{1}}$. If $l_{1} \geq r_{1}$, then using $k=l_{1}$ in Proposition 2.8 and induction we obtain the result. If $l_{1}<r_{1}$, then we consider $y_{a_{2}}$. Notice that $y_{a_{2}}$ cannot be connected to $y_{b_{1}}$ since then $\left\{y_{a_{1}}, y_{a_{2}}, y_{b_{1}}\right\}$ will form a triangle, which is a contradiction since $l \geq 5$. Hence, either $y_{a_{2}}$ is connected to $y_{a_{3}}$, or $y_{a_{2}}$ is connected to $y_{b_{i}}$ for some $i \geq 2$. If $y_{a_{2}}$ is connected to $y_{a_{3}}$, then case (i) yields the result.

Suppose that $y_{a_{2}}$ is connected to $y_{b_{i}}$ for some $i \geq 2$. Without loss of generality, suppose that $i=2$. If $l_{2} \geq r_{2}$, then we may use $k=l_{2}$ in Proposition 2.8 and induction. If $l_{2}<r_{2}$ and since $l_{1}<r_{1}$ we can use Lemma 3.2 and induction.

Finally, for case (iii), suppose that $y_{a_{1}}$ is connected only to $y_{b_{1}}$ and $y_{b_{2}}$. If every $y_{a_{i}}$ is connected to $y_{b_{i_{1}}}$ and $y_{b_{i_{2}}}$ for $i_{1}, i_{2} \neq 1,2$, then either $m=t$, which is impossible since $l$ is odd or there exist $i \neq j$ such that $y_{b_{i}}$ is connected to $y_{b_{j}}$. Then by switching the role of $\alpha$ and $\beta$ we are in either case (i) or case (ii).

We are now ready to state the main theorem of this section.

Theorem 3.4. Let $R$ be a polynomial ring over a field, and let $I$ be a square-free monomial ideal in $R$. We further assume that the line graph of $I$ is the disjoint union of graphs of trees and graphs with a unique odd cycle. Then $I$ is an ideal of linear type.

Proof. The result follows immediately by Proposition 2.8, Proposition 3.1 and Proposition 3.3. 
We conclude this article with the following remark.

Remark 3.5. Let $R$ be a polynomial ring over a field, and let $I$ be a square-free monomial ideal generated by 3 square-free monomials in $R$. Then the fact that $I$ is of linear type is already known, but it also follows immediately from Proposition 3.1 and Proposition 3.3.

Acknowledgments. We wish to thank Ali Alilooee and Yi-Huang Shen for their comments on an earlier version of the manuscript.

\section{REFERENCES}

1. I. Aberbach, L. Ghezzi and H.T. Há, Homology multipliers and the relation type of parameter ideals, Pacific J. Math. 226 (2006), 1-39.

2. L. Busé, Elimination theory in codimension one and applications, INRIA, 5918, June 2006, 1-47.

3. A. Conca and E. De Negri, $M$-sequences, graph ideals, and ladder ideals of linear type, J. Alg. 211 (1999), 599-624.

4. A. Conca, J. Herzog, N.V. Trung and G. Valla, Diagonal subalgebras of bigraded algebras and embeddings of blow-ups of projective spaces, Amer. J. Math. 119 (1997), 859-901.

5. A. Corso and U. Nagel, Monomial and toric ideals associated to Ferrers graphs, Trans. Amer. Math. Soc. 361 (2009), 1371-1395.

6. D. Cox, A. Kustin, C. Polini and B. Ulrich, A study of singularities on rational curves via syzygies, Mem. Amer. Math. Soc. 222 (2013), x+116 pp.

7. D. Cox, J. Little and H. Schenck, Toric varieties, Grad. Stud. Math. 124, American Mathematical Society, Providence, RI, 2011.

8. V. Gasharov, N. Horwitz and I. Peeva, Hilbert functions over toric rings, Michigan Math. J. 57 (2008), 339-357.

9. A. Geramita and A. Gimigliano, Generators for the defining ideal of certain rational surfaces, Duke Math. J. 62 (1991), 61-83.

10. A. Geramita, A. Gimigliano and B. Harbourne, Projectively normal but superabundant embeddings of rational surfaces in projective space, J. Alg. 169 (1994), 791-804.

11. A. Gimigliano, On Veronesean surfaces, Proc. Konin. Ned. Akad. 92 (1989), $75-85$.

12. A. Gimigliano and A. Lorenzini, On the ideal of Veronesean surfaces, Canad. J. Math. 43 (1993), 758-777.

13. H.T. Hà, Box-shaped matrices and the defining ideal of certain blowup surfaces, J. Pure Appl. Alg. 167 (2001), 203-224.

14. On the Rees algebra of certain codimension two perfect ideals, Manuscr. Math. 107 (2002), 479-501. 
15. J. Herzog, A. Simis and W.V. Vasconcelos, Approximation complexes of blowing-up rings, J. Alg. 74 (1982), 466-493.

16. , Approximation complexes of blowing-up rings, II, J. Alg. 82 (1983), $53-83$.

17. J. Herzog, W.V. Vasconcelos and R.H. Villarreal, Ideals with sliding depth, Nagoya Math. J. 99 (1985), 159-172.

18. S. Huckaba, On complete d-sequences and the defining ideals of Rees algebras, Math. Proc. Cambr. Phil. Soc. 106 (1989), 445-458.

19. C. Huneke, Determinantal ideals of linear type, Arch. Math. (Basel) 47 (1986), 324-329.

20. On the symmetric and Rees algebra of an ideal generated by a $d$ sequence, J. Algebra 62 (1980), 268-275.

21. A. Kustin, C. Polini and B. Ulrich, Rational normal scrolls and the defining equations of Rees algebras, J. reine angew. Math. 650 (2011), 23-65.

22. Y-H. Lai, On the relation type of systems of parameters, J. Alg. 175 (1995), $339-358$.

23. K.-N. Lin and C. Polini, Rees algebras of truncations of complete intersections, J. Alg. 410 (2014), 36-52.

24. A. Micali, Sur les algèbres universelles, Ann. Inst. Four. 14 (1964), 33-87.

25. S. Morey, Equations of blowups of ideals of codimension two and three, J. Pure Appl. Alg. 109 (1996), 197-211.

26. S. Morey and B. Ulrich, Rees algebras of ideals with low codimension, Proc. Amer. Math. Soc. 124 (1996), 3653-3661.

27. H. Ohsugi and T. Hibi, Toric ideals generated by quadratic binomials, J. Alg. 218 (1999), 509-527.

28. E. Reyes, C. Tatakis and A. Thoma, Minimal generators of toric ideals of graphs, Adv. Appl. Math. 48 (2012), 64-78.

29. A. Simis, N. Trung and G. Valla, The diagonal subalgebra of a blow-up algebra, J. Pure Appl. Alg. 125 (1998), 305-328.

30. J.A. Soleyman and X. Zheng, Monomial ideals of forest type, Comm. Alg. 40 (2012), 2786-2797.

31. D. Taylor, Ideals generated by monomials in an R-sequence, Ph.D. dissertation, University of Chicago, Chicago, 1966.

32. N.V. Trung, Reduction exponent and degree bound for the defining equations of graded rings, Proc. Amer. Math. Soc. 101 (1987), 229-236.

33. G. Valla, On the symmetric and Rees algebra of an ideal, Manuscr. Math. 30 (1980), 239-255.

34. W. Vasconcelos, Arithmetic of blowup algebras, Lond. Math. Soc. Lect. Note

195, Cambridge University Press, Cambridge, 1994.

35. R.H. Villarreal, Rees algebras of edge ideals, Comm. Alg. 23 (1995), 35133524 . 
36. H-J. Wang, The relation-type conjecture holds for rings with finite local cohomology, Comm. Alg. 25 (1997), 785-801.

Department of Mathematical Sciences, New Mexico State University, Las Cruces, NM 88003

Email address: lfouli@math.nmsu.edu

Academic Affairs, The Pennsylvania State University, Greater Allegheny, MCKeEsport, PA 15132

Email address: kul20@psu.edu 